\title{
The Common Curve of Quadrics Sharing a Self-Polar Simplex (*).
}

\author{
W. L. EdGE (Edimburgh, Gran Bretagna)
}

\begin{abstract}
Summary. - When $n-1$ quadrics in projective space [n] of $n$ dimensions have a common selfpolar simplex their common curve $\Gamma$ admits a group of $2^{\text {th }}$ self-projectivities. The consequent properties of $T$ are investigated, and further specialisations are imposed which amplify the the group and endow $I$ with further properties. There is some reference to the osculating spaces and principal chords of $\Gamma$, and some properties of particular ourves in four and five dimensions are described.
\end{abstract}

\section{I. - Principal properties of the curve.}

1. - A simplex $S$ in a projective space $[n]$ of $n$ dimensions provides $n+1$ involutory projectivities that are mutually commutative and have for product the identity projectivity: namely the harmonic inversions in the vertices and opposite bounding primes of $S$. When $S$ is taken as simplex of reference for a system of homogeneous coordinates $x_{j}$ then $h_{j}$, associated with the vertex $X_{j}$ and the opposite bounding prime $x_{j}=0$, multiplies the single coordinate $x_{j}$ by -1 and leaves the other $n$ coordinates unchanged. That $h_{j}$ is an involution, that the different $h_{j}$ commute, and that

$$
h_{0} h_{1} \ldots h_{n}=I \text {, }
$$

are clear from the representation of the $h_{j}$ as diagonal matrices. These $h_{j}$ generate an elementary abelian group $E$ of $2^{n}$ projectivities. The [s-1] spanned by any $s$ vertices of $S$ and the $[n-s]$ spanned by the other $n+1-s$ vertices are a pair of fundamental spaces, or axes, of one of the $2^{n}-1$ involutions in $E$.

Any quadric $\Omega$ for which $S$ is self-polar is invariant under $E$; each point of $\Omega$ that is not in any face of $S$, i.e. for which no $x_{j}$ is zero, is one of a batch $B$ of $2^{n}$ points of $\Omega$ that is invariant under $E$. The same is true of the manifold common to any set of such quadrics. If there are $n-1$ quadrics in the set this manifold is, in general, an irreducible curve $\Gamma_{n}$ of order $2^{n-1}$. Since it is the complete intersection of $n-1$ primals its genus $\pi_{n}$ is deducible from the known fact that the canonical series is cut on $\Gamma_{n}$ by primals of order $2(n-1)-(n+1)=n-3$, so that

$$
\begin{aligned}
2 \pi_{n}-2 & =2^{n-1}(n-3) \\
\pi_{n} & =2^{n-2}(n-3)+1 .
\end{aligned}
$$

as statied on p. 185 of [1].

(*) Entrata in Redazione il 23 giugno 1976.

16 - Annali di Matematica 
2. $-\Gamma_{n}$ is determined by $n-1$ simultaneous equations

$$
\Omega_{k} \equiv \sum \alpha_{j}^{(k)} x_{j}^{2}=0 \quad k=0,1, \ldots, n-2
$$

where summation is over $j$ and runs, unless otherwise stated, from 0 to $n$. It is presumed that (2.1), regarded as linear equations in $x_{3}^{2}$, are linearly independent; the matrix $\left[\alpha_{j}^{(k)}\right]$ has full rank $n-1$. This implies, since all $(n-1)$ rowed determinants are non-zero, that $\Gamma_{n}$ is skew to every bounding [n-2] of $S$ : no two of the $n+1$ coordinates can be zero simultaneously on $\Gamma_{n}$. A point of $\Gamma_{n}$ in $x_{i}=0$ is invariant under $h_{j}$ and the batch determined by it consists not of $2^{n}$ but of only $2^{n-1}$ points; they compose the whole intersection of $\Gamma_{n}$ and $x_{j}=0$ and may be called a critical batch $B_{j}^{*}$.

There is a linear combination of the $n-1$ equations (2.1) from which any $n-2$ of the squares $x_{j}^{2}$ are absent, but it is not possible so to eliminate $n-1$ of the $n+1$ squares. Among the quadrics through $\Gamma_{n}$ are cones whose vertices are the bounding $[n-3]$ 's opposite to the plane faces of $S$, but $\Gamma_{n}$ does not lie on any cone with an $[n-2]$ for vertex.

The invariance of $\Gamma_{n}$ under the $h_{f}$ discloses the special character of the points in the $n+1$ critical batches.

The tangents of $\Gamma_{n}$ at all the points in $B_{j}^{*}$ concur at $X_{j}$. If $P P_{j}$ is a chord of $\Gamma_{n}$ through $X_{j}$ and $P$ tends along $\Gamma_{n}$ to a point in $B_{j}^{*}$ then $P_{j}$, on the line $X_{j} P$, tends simultaneously to this same point; the osculating plane there has 4-point intersection with $\Gamma_{n}$. Repetition of the argument proves that the osculating $[s]$ has $2 s$-point intersection for $s=1,2, \ldots, n-1$. These points, $2^{n-1}$ in each of the $n+1$ bounding primes of $s$, may be appropriately called stalls of $\Gamma_{n}$. The branch centred at a stall is characterized by the integers

$$
\alpha_{0}=\alpha_{1}=1, \quad \alpha_{2}=\alpha_{3}=\ldots=\alpha_{n-1}=2 .
$$

For a definition of such integers see [13], p. 28 where they are designated as $v, v^{\prime}$, $v^{\prime \prime}, \ldots$; or $[8]$, p. 246 where Segre's $i_{s}$ is $\alpha_{0}+\alpha_{1}+\ldots+\alpha_{s}$.

The $2^{n}$ points of a batch are in perspective from each of the $n+1$ vertices of $S$. The chords of $\Gamma_{n}$ through $X_{3}$ generate a two-dimensional cone of order $2^{n-2}$; all points on these chords satisfy $n-3$ linearly independent equations obtained by eliminating $x_{j}^{2}$ from (2.1). But these, $x_{j}^{2}$ being absent, are equations, one less in number, of the same form and involving one coordinate less: the chords of $\Gamma_{n}$ through $X_{j}$ meet $x_{j}=0$ in a curve $\Gamma_{n-1}$. There is a $(2,1)$ correspondence between $\Gamma_{n}$ and this projection, branching at the $2^{n-1}$ points of $B_{j}^{*}$. An application of Zeuthen's formula ([12], p. 152, [13], p. 223, [8] p. 83, [10] p. 211) allows $\pi_{n}$ to be calculated by recurrence, the initial condition being $\pi_{3}=1$ for the elliptic quartic, or even $\pi_{2}=0$ for a conic. The calculation yields the same value for $\pi_{n}$ as in $\S 1$, and has already been used on p. 338 of $[6]$. 
3. - There is a representation of $\Gamma_{n}$ that proves to be convenient when investigating the geometry of the curve.

Regard the coefficients $\alpha^{(k)}$ as the homogeneous coordinates $y^{(k)}$ of $n+1$ points $A_{j}$ in a projective space $[n-2]$. Through these points there passes a unique rational normal curve $C$. Choose a coordinate system in $[n-2]$ so that $O$ has the standard parametric form $y^{(k)}=t^{k}(k=0,1, \ldots, n-2)$; the points $A_{j}$ will have for their parameters $n+1$ unequal numbers $a_{j}$. In order to avoid an infinite value, and so ensure that every $A_{j}$ has its first coordinate $y^{(0)}$ non-zero, one has only to arrange that the contact of $C$ with its osculating $[n-3] y^{(0)}=0$ is not at any of the $A_{j}$. Then, $A_{i}$ being $\left(1, a_{j}, a_{j}^{2}, \ldots, a_{j}^{n-2}\right), \Gamma_{n}$ is given by

$$
\Omega_{k} \equiv \sum a_{j}^{k} x_{j}^{2}=0 \quad(k=0,1, \ldots, n-2)
$$

where $k$ is now an actual power and not a mere superseript. There is a practical illustration of this circumstance when $n=4$ in [3].

The equations (3.1) show that $\Gamma_{n}$ depends only on the $n-2$ projective invariants of the $n+1$ numbers $a_{j} ; \Gamma_{n}$ has only $n-2$ moduli whereas a general non-hyperelliptic curve of genus $\pi_{n}$ has $3 \pi_{n}-3=2^{n-2}(3 n-9)$. The specialisation is due to the invariance under $E$. Since $\Gamma_{n}$ is projected from a bounding [ $s$ ] of $S$ onto the opposite $[n-s-1]$ into a $\Gamma_{n-s-1}$ covered $2^{s+1}$ times it is in multiple correspondence with curves of lower genera. If $n=4$, say, $\Gamma_{4}$ is a special canonical curve of genus 5 with only 2 moduli whereas the general curve of genus 5 has 12 moduli. $\Gamma_{4}$ is projected from each of the 5 vertices of $S$ into an elliptic quartic, covered twice, with eight branch points; it is projected from each of the ten edges of $S$ into a plane conic covered four times.

4. - Write, now

$$
f(\theta) \equiv \prod_{j=0}^{n}\left(\theta-a_{j}\right) \equiv \sum_{p=0}^{n+1}(-1)^{p} e_{p} \theta^{n-p+1}
$$

and

$$
s_{k}=\sum_{=0}^{n} a_{j}^{k} / f^{\prime}\left(a_{j}\right)
$$

Then, as is seen from the decomposition into partial fractions of $\theta^{k} / f(\theta)$, or otherwise,

$$
s_{0}=s_{1}=\ldots=s_{n-1}=0, \quad s_{n}=1 .
$$

So two solutions, and therefore the only two so far as linear dependence is concerned, of (3.1) are

$$
x_{j}^{2}=1 / f^{\prime}\left(a_{j}\right), \quad x_{j}^{2}=a_{j} / f^{\prime}\left(a_{j}\right)
$$

and the general solution is

$$
x_{j}^{2}=\left(\theta+a_{j}\right) / f^{\prime}\left(a_{i}\right) \quad j=0,1, \ldots, n .
$$


This is a parametric form not, of course, for $\Gamma_{n}$ but for a batch $B(\theta)$ on $\Gamma_{n}$; the $2^{n}$ different points of $B(\theta)$ answer to the different signing of the $n+1$ square roots of the $x_{j}^{2}$. The critical batch $B_{j}^{*}$ of stalls is $B\left(-a_{j}\right)$.

The value of $s_{N}$ for $N>n$ can be found by using initial conditions (4.1) and the recurrence relation

$$
s_{N}-e_{1} s_{N-1}+\ldots+(-1)^{n+1} e_{n+1} s_{N-n-1}=0
$$

so that, to give the simplest example, $s_{n+1}=e_{1} s_{n}=e_{1}$. The $x_{j}^{2}$, as parametrised in (4.3), thus satisfy

$$
\Omega_{n-1}=1, \quad \Omega_{n}=0+e_{1},
$$

and $B(\theta)$ is the intersection of $\Gamma_{n}$ with the quadric $\Omega_{n}=\left(\theta+e_{1}\right) \Omega_{n-1}$. It will be found convenient to refer occasionally to the quadrics $\Omega_{n}=0$ and $\Omega_{n-1}=0$ which with the $n-1$ quadrics (3.1), compose a linearly independent set of $n+1$ linear combinations of the $n+1$ squares $x_{j}^{2}$.

This parametrisation of batches on $\Gamma_{n}$ really goes as far back, for $n=3$, as SALMON ([7], p. 195). The explicit form (4.3) in [n] is given by BaKaiR ([1], p. 185) who exploits it to advantage for $n=4$ in his account of Segre's cyclide, and for $n=\mathbf{5}$ in his geometrical treatment of the line geometry of Kummer's surface ([1], pp. 218 et seq.).

5. - If an algebraic curve $\Gamma$ in $[n]$ has genus $p$, order $m$-the number of its intersections with a prime-and class $m^{\prime}$-the number of its osculating primes through a point-then it is known ([9], p. 86) to have

$$
\frac{1}{2}(m-n)(m-n-1)+\frac{1}{2}\left(m^{\prime}-n\right)\left(m^{\prime}-n-1\right)-n(n+1) p
$$

principal chords, a chord being principal when it lies in the osculating primes at both its intersections with $\Gamma$.

As with so much successful enumerative work the procedure by which the number is derived gives no information about how these chords may be grouped, or about the geometry within such a group or the relations of such groups to one another. These matters are likely to be relevant when $\Gamma$ has special properties: for example, any projectivity under which $\Gamma$ is invariant must permute the principal chords among themselves.

The order $2^{n-1}$ and genus $\pi_{n}$ of $\Gamma_{n}$ have been given already; its class $2^{n-1}(2 n-3)$ was found by elementary methods in [5] and can also be found in other ways. It follows that the number of principal chords of $\Gamma_{n}$ is

$$
2^{2 n-2}\left(2 n^{2}-6 n+5\right)-2^{n-2}\left(n^{3}+2 n^{2}-5 n-2\right) .
$$


This accords with the normal elliptic quartic having 24 principal chords; this fact is well known as a special case of the number for the normal elliptic curve of any order, and has also been established independently in [4] by reasoning allied to that to be used now.

There will be two kinds of principal chords of $\Gamma_{n}$ : such a chord may join points in the same batch, or it may not. If it does it is invariant under that involution in $E$ which transposes these two points in the batch, and so meets a pair of opposite bounding spaces of $S$. For example: if $n=5$ such a chord may

(i) pass through a vertex $X_{j}$ and meet $x_{j}=0$, or

(ii) meet an edge and opposite solid of $S$, or

(iii) meet an opposite pair of plane faces of $S$.

In (i) the "chord" is the tangent at a stall. This, in view of the high multiplicity $2 n-2$ of the intersection of $\Gamma_{n}$ with its osculating prime at the stall, raises the question: what is the number $\mu_{n}$ of times that the tangent at a stall is to be reckoned among the principal chords?

There will also be principal chords joining points in different batches. Should the points be in batehes $B_{1}$ and $B_{2}$ the osculating prime at any point of $B_{1}$ contains a point of $B_{2}$ at which the osculating prime, in turn, contains the point of $B_{1}$. Such "transversal" principal chords occur in sets of $2^{n}$.

6. - The osculating prime of $\Gamma_{n}$ at $x=\xi$ is, by equation (3.1) on p. 40 of [5],

$$
\sum\left\{f^{\prime}\left(a_{j}\right)\right\}^{n-2} \xi_{j}^{2 n-3} x_{j}=0
$$

or, alternatively, if $\xi$ belongs to $B(\theta)$,

$$
\sum\left(\theta+a_{j}\right)^{n-2} \xi_{j} x_{j}=0 .
$$

If, then, $\xi$ belongs to $B(\theta)$ and $\eta$ to $B(\varphi)$ the chord $\xi \eta$ is principal if, and only if,

$$
\sum\left(\theta+a_{j}\right)^{n-\frac{3}{2}}\left(\varphi+a_{j}\right)^{\frac{1}{2}} / f^{\prime}\left(a_{j}\right)=0=\sum\left(\theta+a_{j}\right)^{\frac{1}{2}}\left(\varphi+a_{j}\right)^{n-\frac{8}{2}} / f^{\prime}\left(a_{j}\right) .
$$

Both these equations are unchanged if the signs of $\sqrt{\left(\theta+a_{j}\right)}$ and $\sqrt{\left(\varphi+a_{3}\right)}$ are both changed; hence a chord $\xi \eta$ that is principal is accompanied by other principal chords, one through each point of the batch to which $\xi$ belongs.

If the intersections of $\Gamma_{n}$ with a principal chord both belong to $B(\theta)$ then, for some selection of signs,

$$
\sum \pm\left(\theta+a_{j}\right)^{n-1} / f^{\prime}\left(a_{j}\right)=0 .
$$

Now this sum would be zero identically in $\theta$ by (4.1) were all signs the same; hence 
both sums of terms that are signed positively and of terms that are signed negatively, are zero: say, for convenience,

$$
\sum_{0}^{p}\left(\theta+a_{j}\right)^{n-1} / f^{\prime}\left(a_{j}\right)=0=\sum_{p+1}^{n}\left(\theta+a_{j}\right)^{n-1} / f^{\prime}\left(a_{j}\right) .
$$

These two sums are negatives of each other, and the two equations have the same $\operatorname{roots} \theta_{1}, \theta_{2}, \ldots, \theta_{n-1}$ : Now

$$
\sum_{0}^{p}\left(\theta+a_{j}\right)^{n-1} / f^{\prime}\left(a_{j}\right)=\sum_{0}^{p}\left\{f^{\prime}\left(a_{j}\right)\right\}^{n-2} \xi_{j}^{2 n-2} .
$$

When $\theta$ is assigned each $\xi_{j}$ has either of two values, so that the sum on the right is, for each root $\theta_{j}$, zero for $2^{p}$ points $\xi$ in $x_{p+1}=x_{p_{+2}}=\ldots=x_{n}=0$. Likewise there are $2^{n-p-1}$ in the opposite $[n-p-1]$ spanned by the vertices $X_{p+1}, X_{p+2}, \ldots, X_{n}$ of $S$. Thus each of the $n-1$ roots $\theta_{j}$ provides $2^{n-1}$ principal chords joining pairs of points in $B\left(\theta_{j}\right)$. Take $p$ over the range $1 \leqslant p \leqslant n-2$ so that each such chord is included twice. The number of principal chords so accounted for is

$$
\frac{1}{2}(n-1) \cdot 2^{n-1}\left\{\left(\begin{array}{c}
n+1 \\
2
\end{array}\right)+\left(\begin{array}{c}
n+1 \\
3
\end{array}\right)+\ldots+\left(\begin{array}{c}
n+1 \\
n-1
\end{array}\right)\right\}=2^{n-1}(n-1)\left(2^{n}-n-2\right)
$$

If, in particular, $n=3$ one obtains 24 principal chords; each of the three pairs of opposite edges of the tetrahedron $S$ is associated with two batches on $\Gamma_{3}$, and each of these batches has its eight points joined in pairs by four principal chords all transversal to the pair of opposite edges. These facts agree with the findings in [4].

Incidentally, as these are all the principal chords of $\Gamma_{3}, \mu_{3}=0$.

The preceding discussion provides 240 principal chords of $\Gamma_{4}, 24$ transversal to any edge and opposite plane face of the simplex $S$. This edge and face are thus associated with three batches $B\left(\theta_{1}\right), B\left(\theta_{2}\right), B\left(\theta_{3}\right)$. Each $\theta_{i}$ yields a pair of points on the edge that are harmonic to the vertices of $S$ as well as four points in the face forming a quadrangle with the vertices of $S$ for its triangle of diagonal points; the eight joins of the two points on the edge to the four points in the face are all principal chords of $\Gamma_{4}$, and join points of $B\left(\theta_{i}\right)$ in pairs.

$\Gamma_{4}$ has, by (5.1), 536 principal chords in all so that 296 remain to be accounted for. But 296 is not divisible by $2^{4}$ so that the tangents at the 40 stalls of $\Gamma_{4}$ must be included, and that an odd number of times; $\mu_{4}$ is not zero, and is odd.

The number in (5.1) exceeds that in (6.3) by

$$
2^{2 n-2}\left(2 n^{2}-8 n+7\right)-2^{n-2}\left(n^{3}-7 n+2\right) .
$$

If one subtracts from this the number of stalls counted $\mu_{n}$ times the residue is the number of principal chords joining points of different batches; this has to be a multiple of $2^{n}$. But the residue is

$$
2^{2 n-2}\left(2 n^{2}-8 n+7\right)-2^{n-2}\left\{(n-1)^{2}(n+2)-4 n\right\}-(n+1) 2^{n-1} \mu_{n} .
$$


This, whatever the integer $\mu_{n}$, is divisible by $2^{n}$ whenever $n$ is odd. If, however, $n$ is even then

$$
\begin{aligned}
& \mu_{n} \text { is even if } n \equiv 2(\bmod 4), \\
& \mu_{n} \text { is odd if } n \equiv 0(\bmod 4) .
\end{aligned}
$$

Questions of multiplicity can present awkward problems, and the value of $\mu_{n}$ is not the only one. Suppose, as does happen upon occasion (see $\$ 18,21$ below), that a principal chord $\xi \eta$ of $\Gamma_{n}$ lies not only in the osculating primes at both $\xi$ and $\eta$ but also in the osculating spaces of dimensions $n-2, n-3, \ldots, n-s$ at both points. How often must such a chord be reckoned? For how many principal chords does it account?

7. - There is a special situation, instances of which are to be encountered later, in which principal chords can be detected joining points in $B(\infty)$ and joining points in $B(0)$. It occurs when $n+1$ is composite and $\Gamma_{n}$ invariant under a regular permutation of the coordinates; say $n+1=g h$ with the coordinates permuted in $g$ cycles of $h$ :

$$
\left(x_{0} x_{g} \ldots x_{g(h-1)}\right) \ldots\left(x_{g-1} x_{2 g-1} \ldots x_{h g-1}\right)
$$

The reason why $B(\infty)$ and $B(0)$ obtrude is that they are both invariant under the imposed projectivity o whereas all other batches are permuted in cycles of $h$.

This situation occurs if

$$
\Omega_{k}=\sum_{j=0}^{g-1} a_{j}^{k}\left\{x_{j}^{2}+\varepsilon^{k} x_{j+g}^{2}+\varepsilon^{2 k} x_{j+2 g}^{2}+\ldots+\varepsilon^{(h-1) k} x_{j+(h-1) g}^{2}\right\}
$$

with $\varepsilon$ a primitive $h$-th root of unity. The effect of $\wp$ is to multiply $\Omega_{k}$ by $\varepsilon^{-k}$ and so leave each quadric $S_{k}=0$ invariant. Then

$$
f(\theta) \equiv\left(\theta^{h}-a_{0}^{h}\right)\left(\theta^{h}-a_{1}^{h}\right) \ldots\left(\theta^{h}-a_{g-1}^{h}\right) \equiv \psi\left(\theta^{h}\right)
$$

say so that $f^{\prime}(\theta)=h \theta^{h-1} \psi^{\prime}\left(\theta^{h}\right)$ whence it follows that

$$
f^{\prime}\left(a_{j}\right): f^{\prime}\left(\varepsilon a_{j}\right): \ldots: f^{\prime}\left(\varepsilon^{h-1} a_{j}\right)=1: \varepsilon^{-1}: \varepsilon^{-2}: \ldots: \varepsilon^{1-h}
$$

This shows that the terms of either sum obtained by taking $\theta=\infty$ or 0 in (6.1) fall into $g$ sets of $h$, the sum of any set being zero. For, $\lambda$ being some constant,

$$
\sum_{r=0}^{h-1} 1 / f^{f}\left(\varepsilon^{r} a_{j}\right)=\lambda \sum_{0}^{h-1} \varepsilon^{r}=0
$$

and

$$
\sum_{r=0}^{h-1} \frac{\left(\varepsilon^{r} a_{j}\right)^{g h-2}}{f^{\prime}\left(\varepsilon^{r} a_{j}\right)}=\left(a_{j}\right)^{g h-2} \sum_{0}^{h-1} \frac{1}{\varepsilon^{2 r} f^{\prime}\left(\varepsilon^{r} a_{j}\right)}=\lambda a_{j}^{g h-2} \sum_{0}^{h-1} \varepsilon^{-r}=0 .
$$




\section{II. - Curves invariant under additional self-projectivities of period 2 or 3.}

8. $-\Gamma_{n}$ can be specialised to admit ampler groups of self-projectivities than $E$ and so have still fewer moduli.

If $n=2 p+1$ is odd a specialisation suggests itself at once; choose the $2 p+2$ numbers $a_{j}$ to be $p+1$ pairs of an involution $I$ (this is, of course, no specialisation if $p=1$ ). There is no loss of generality in assigning parameters 0 and $\infty$ to the foci of $I$; then the parameters of any pair of $I$ sum to zero: say $a_{j}+a_{j_{+p+1}}=0$, and $\Gamma_{n}^{\prime}$ is the common curve of the $2 p$ quadrics

$$
\Omega_{k} \equiv \sum_{j=0}^{p} a_{j}^{k}\left\{x_{j}^{2}+(-1)^{k} x_{j+p+1}^{2}\right\}=0 \quad k=0,1, \ldots, 2 p-1
$$

It is invariant not only under $E$ but also under the $(p+1)$-fold transposition.

$$
\left(x_{0} x_{p+1}\right)\left(x_{1} x_{p+2}\right) \ldots\left(x_{p} x_{2 p+1}\right)
$$

of the $2 p+2$ coordinates in pairs; for this leaves $\Omega_{k}$ unchanged when $k$ is even, changes $\Omega_{k}$ into $-\Omega_{k}$ if $k$ is odd. The corresponding projectivity is harmonic inversion $J$ in the skew pair of $[p]$ 's.

$$
\left\{\begin{array}{l}
x_{0}-x_{p+1}=x_{1}-x_{p+2}=\ldots=x_{p}-x_{2 p+1}=\mathbf{0}, \\
x_{0}+x_{p+1}=x_{1}+x_{p+2}=\ldots=x_{p}+x_{2 p+1}=\mathbf{0} .
\end{array}\right.
$$

Both these $[p]$ 's lie on the $p$ quadries

$$
\Omega_{1}=0, \quad \Omega_{3}=0, \ldots, \Omega_{2 p-1}=0 ;
$$

indeed (8.3) all hold whenever

$$
x_{0}^{2}-x_{p+1}^{2}=x_{1}^{2}-x_{p+2}^{2}=\cdots=x_{p}^{2}-x_{2 p+1}^{2}=0,
$$

a set of $p+1$ equations representing $2^{p+1}[p]$ 's composed of $2^{p}$ skew pairs of opposites of which (8.2) is but one. Each pair affords a harmonic inversion leaving $\Gamma_{n}^{\prime}$ invariant. These skew pairs are of course obtainable from (8.2) by using the $h_{j}$ of $\S 1$. Since the $2^{2 p+1}$ operations of $E$ permute the $2^{p}$ pairs among themselves one expects each pair to be invariant under a group of $2^{p+1}$ operations of $E$. For example: each member of (8.2) is unchanged by any of

$$
h_{0} h_{p+1}, h_{1} h_{p+2}, \ldots, h_{p} h_{2 p+1}
$$


which, commuting and having product identity, generate an elementary abelian group of order $2^{p}$; this is amplified to one of order $2^{p+1}$ by adjoining

$$
h_{0} h_{1} \ldots h_{p} \equiv h_{p++1} h_{p+2} \ldots h_{2 p+1}
$$

transposing the two $[p]$ 's of $(8.2)$.

It will not have escaped notice that just as (8.3) hold in consequence of (8.4), so

$$
\Omega_{0}=0, \quad \Omega_{2}=0, \ldots, \Omega_{2 p-2}=0
$$

hold in consequence of

$$
x_{0}^{2}+x_{y+1}^{2}=x_{1}^{2}+x_{p+2}^{2}=\ldots=x_{p}^{2}+x_{2 p+1}^{2}=0 .
$$

This is a second set of $2^{p}$ skew pairs of opposite $[p]^{\prime} \mathrm{s}$, and each pair is invariant under the same group of order $2^{p+1}$.

Each of the $2^{p}$ pairs in (8.4) is associated with a pair in (8.6). To explain this geometrically take (8.2). The equations ( $\lambda$ a parameter)

$$
x_{0} / x_{p_{+1}}=x_{1} / x_{p_{+2}}=\ldots=x_{p} / x_{2 p+1}(=\lambda)
$$

represent a singly-infinite set of $[p]$ 's, all skew to one another, generating a locus $V$ of dimension $p+1$, indeed the "Segre product " $[p] \times[1]$ mentioned on p. 174 of [8]. For $\lambda= \pm 1$ one has the pair (8.2); for $\lambda= \pm i$ one has the pair

$$
\left\{\begin{array}{l}
x_{0}-i x_{p+1}=x_{1}-i x_{p+2}=\ldots=x_{p}-i x_{p p+1}=0 \\
x_{0}+i x_{p+1}=x_{1}+i x_{x+2}=\ldots=x_{p}+i x_{2 p+1}=0
\end{array}\right.
$$

of (8.6). The harmonic inversions in (8.2) and (8.7) commute, their product being the inversion in those $[p]$ on $V$ having $\lambda=0, \infty$, i.e. in

$$
x_{0}=x_{1}=\ldots=x_{p}=0 \quad \text { and } \quad x_{p+1}=x_{p_{+2}}=\ldots=x_{2 p+1}=0,
$$

so that this product is just $h_{0} h_{1} h_{2} \ldots h_{p}$.

9. - After observing that any of the $2^{p+1}$ skew pairs of [p]'s can be transformed into any other by a projectivity leaving $\Gamma_{n}^{\prime}$ invariant it is sufficient to consider any one pair.

Since both $[p]$ lie on $p$ of the $2 p$ quadries defining $\Gamma_{n}^{\prime}$ the $2^{p}$ common points of the quadric $(p-1)$-folds in which the remaining $p$ quadrics meet either $[p]$ are on $\Gamma_{n}^{\prime}$. Now every point of $\Gamma_{n}^{\prime}$ is paired with another in $J$, and the chords jointng such pairs, all being transversal to both $[p]$ 's, generate a scroll $R$, of order $\mu$ say, meeting the 
two $[p]$ 's in directrix curves, of order $m$ say (the curves have the same order since the $[p]$ 's, say the pair (8.2), in which they lie are transformed into each other by harmonic inversion in the pair (8.7)). Among the generators of $R$ those through the intersections of $\Gamma_{n}^{\prime}$ with the two [p]'s are tangents of $\Gamma_{n}^{\prime}$.

The genus $\pi$ of $R$ can be found at once by applying Zeuthen's formula. For every directrix (say a prime section) of $R$ is in $(1,2)$ correspondence with $\Gamma_{n}^{\prime}$, the coincidences on $\Gamma_{n}^{\prime}$ being its $2^{p+1}$ contacts with generators of $R$; hence the formula gives

$$
\begin{aligned}
4(\pi-1)+2^{p+1} & =2\left(\pi_{n}-1\right)=2^{2 p}(2 p-2) \\
\pi & =1+(p-1) 2^{2 p-2}-2^{p-1} .
\end{aligned}
$$

A prime meets $\Gamma_{n}^{\prime}$ in $2^{2 p}$ points; if the prime contains either [p] these consist of the $2^{p}$ points of $\Gamma_{n}^{t}$ in $[p]$ and two on each of those $m$ generators of $R$ that pass through the $m$ intersections of the prime with the directrix in the opposite $[p]$; so

$$
\begin{aligned}
& 2^{s p}=2^{p}+2 m, \\
& m=2^{2 p-1}-2^{p-1},
\end{aligned}
$$

And since the prime meets $R$ in the directrix of order $m$ and $m$ generators

$$
\mu=2 m=2^{3 p}-2^{p}
$$

There are $2^{p+1}$ such scrolls containing $\Gamma_{n}^{\prime}$.

10. - The discussion in $\S 7$ shows, with $h=2$ and $g=p+1$, that principal chords of $\Gamma_{n}^{\prime}$ join points in $B(\infty)$ as well as in $B(0)$. Since $f(\theta)$ is here an even, and so $f^{\prime}(\theta)$ an odd, function, $f^{\prime}\left(a_{j}\right)=-f^{\prime}\left(-a_{i}\right)$; thus both sums

$$
1 / f^{\prime}\left(a_{j}\right)+1 / f^{\prime}\left(-a_{j}\right) \text { and } a_{j}^{2 p} / f^{\prime}\left(a_{j}\right)+\left(-a_{j}\right)^{2 p} / f^{\prime}\left(-a_{j}\right)
$$

are zero and the $2 p+2$ terms in (6.1) fall now, should $\theta$ be either 0 or $\infty$, into $p+1$ pairs with each pair summing to zero. So one obtains, in either batch, $2^{2 p}$ principal chords transversal to an edge $X_{j} X_{j+p+1}$ and opposite $[2 p-1]$ of $\mathbb{S}, 2^{2 p}$ transversal to a solid $X_{j} X_{j+v+1} X_{k} X_{k+x+1}$ and opposite [2p-3] of $S$, and so on. Thus, among the joins of either $B(\infty)$ or $B(0)$ there are

$$
2^{2 p} \cdot \frac{1}{2}\left\{\left(\begin{array}{c}
p+1 \\
1
\end{array}\right)+\left(\begin{array}{c}
p+1 \\
2
\end{array}\right)+\ldots+\left(\begin{array}{c}
p+1 \\
p
\end{array}\right)\right\}=2^{3 p}-2^{2 p}
$$

principal chords. Since there are $2^{2 \mathfrak{p}+1}$ points in a batch one expects there to be

$$
2\left(2^{3 p}-2^{2 p}\right) / 2^{2 p+1}=2^{p}-1
$$


prineipal chords through each; indeed both $B(0)$ and $B(\infty)$ are partitioned into $2^{p+3}$ subsets of $2^{p}$ points, the join of any two points in the same subset being a principal chord. Each subset is an orbit under the group, of order $2^{p}$, generated by the operations (8.5).

One now proceeds to illustrate these properties of $\Gamma_{2 p+1}^{\prime}$, by describing the figure in [5].

11. $-\Gamma_{5}^{\prime}$, with $p=2$, has order 16 and genus 17 ; it lies on eight scrolls of order 12 each having two plane sextics of genus 7 for directrices.

$\Gamma_{5}^{\prime}$ is the common curve of the four quadrics

$$
\left\{\begin{aligned}
x_{0}^{2}+x_{3}^{2}+x_{1}^{2}+x_{4}^{2}+x_{2}^{2}+x_{5}^{2} & =0, \\
a\left(x_{0}^{2}-x_{3}^{2}\right)+b\left(x_{1}^{2}-x_{4}^{2}\right)+c\left(x_{2}^{2}-x_{5}^{2}\right) & =0, \\
a^{2}\left(x_{0}^{2}+x_{3}^{2}\right)+b^{2}\left(x_{1}^{2}+x_{4}^{2}\right)+c^{2}\left(x_{2}^{2}+x_{5}^{2}\right) & =0, \\
a^{3}\left(x_{0}^{2}-x_{3}^{2}\right)+b^{3}\left(x_{1}^{2}-x_{4}^{2}\right)+c^{3}\left(x_{2}^{2}-x_{5}^{2}\right) & =0 .
\end{aligned}\right.
$$

Any of the eight planes

$$
x_{0}^{2}+x_{3}^{2}=x_{1}^{2}+x_{4}^{2}=x_{2}^{2}+x_{5}^{2}=0
$$

meets $\Gamma_{5}^{\prime}$ in the four points for which

$$
a x_{0}^{2}+b x_{1}^{2}+c x_{2}^{2}=a^{3} x_{0}^{2}+b^{3} x_{1}^{2}+c^{3} x_{2}^{2}=0 ;
$$

these 32 points will be shown to be $B(\infty)$. Any of the eight planes

$$
x_{0}^{2}-x_{3}^{2}=x_{1}^{2}-x_{4}^{2}=x_{2}^{2}-x_{5}^{2}=0
$$

meets $\Gamma_{5}^{\prime}$ in the four points for which

$$
x_{0}^{2}+x_{1}^{2}+x_{2}^{2}=a^{2} x_{0}^{2}+b^{2} x_{1}^{2}+c^{2} x_{2}^{2}=0 ;
$$

these 32 points compose $B(0)$.

Since, here,

$$
f(\theta) \equiv\left(\theta^{2}-a^{2}\right)\left(\theta^{2}-b^{2}\right)\left(\theta^{2}-c^{2}\right)
$$

it follows that

$$
\left(b^{2}-c^{2}\right) f^{\prime}(a) / a=\left(c^{2}-a^{2}\right) f^{\prime}(b) / b=\left(a^{2}-b^{2}\right) f^{\prime}(c) / c
$$

and these relations, coupled with the fact of $f^{\prime}(\theta)$ being an odd function, give the parametric form for $B(\theta)$, namely, by (4.3),

$$
\xi_{0}^{2}=\left(b^{2}-e^{2}\right)(\theta+a) / a, \quad \xi_{3}^{2}=-\left(b^{2}-c^{2}\right)(\theta-a) / a
$$


with the equations obtained from these by imposing simultaneously the cyclic permutations $(a b c),(012),(345)$. So $B(0)$ and $B(\infty)$ are as stated.

The osculating [4] of $\Gamma_{5}^{\prime}$ at a point $\xi$ of $B(\infty)$ is, by (3.1) of [5], $\sum \xi_{j} x_{j}=0$; it clearly contains $\xi$ itself by (11.2). But so do, for the same reason, the three [4]'s

$$
\xi_{0} x_{0}+\xi_{3} x_{3}=0, \quad \xi_{1} x_{1}+\xi_{4} x_{4}=0, \quad \xi_{2} x_{2}+\xi_{5} x_{5}=0 .
$$

Hence the osculating [4] at any of the points

$$
\begin{array}{rrrrrr}
\xi_{0} & \xi_{1} & \xi_{2} & \xi_{3} & \xi_{4} & \xi_{5} \\
-\xi_{0} & \xi_{1} & \xi_{2} & -\xi_{3} & \xi_{4} & \xi_{5} \\
\xi_{0} & -\xi_{1} & \xi_{2} & \xi_{3} & -\xi_{4} & \xi_{5} \\
\xi_{0} & \xi_{1} & -\xi_{2} & \xi_{3} & \xi_{4} & -\xi_{5}
\end{array}
$$

of $B(\infty)$ contains all three others. The 32 points of $B(\infty)$ fall into 8 tetrahedra, the edges of the tetrahedra being principal chords. The vertices of a tetrahedron are obtained from any one of them by harmonie inversions in

$$
\begin{aligned}
& \text { the edge } X_{0} X_{3} \text { and opposite bounding solid of } S \text {, } \\
& \ldots \ldots, x_{1} X_{4} \ldots \ldots \ldots \ldots \ldots \ldots \ldots \ldots \ldots, \\
& \cdots \cdots \cdots X_{2} X_{5} \ldots \ldots \ldots \ldots \ldots \ldots \ldots \cdots \cdots
\end{aligned}
$$

The analogous situation holds with $B(0)$; at a point $\xi$ of this batch the osculating [4], is, again by (3.1) of [5],

$$
a^{8}\left(\xi_{0} x_{0}-\xi_{3} x_{3}\right)+b^{a}\left(\xi_{1} x_{1}-\xi_{4} x_{4}\right)+c^{3}\left(\xi_{2} x_{2}-\xi_{5} x_{5}\right)=0
$$

and one now has the three [4]'s

$$
\xi_{0} x_{0}=\xi_{3} x_{3}, \quad \xi_{1} x_{1}=\xi_{4} x_{4}, \quad \xi_{2} x_{2}=\xi_{5} x_{5}
$$

all containing $\xi$ by (11.4).

12. - The chords of $\Gamma_{5}^{\prime}$ which join pairs of points harmonic inverses of each other in the planes

$$
x_{0}-x_{3}=x_{1}-x_{4}=x_{2}-x_{6}=0 \text { and } \quad x_{0}+x_{3}=x_{1}+x_{4}=x_{2}+x_{5}=0
$$

generate a scroll $R$ with a directrix in each plane. The pairs on $\Gamma_{5}^{\prime}$ are

$$
\left(x_{0}, x_{1}, x_{2}, x_{3}, x_{1}, x_{5}\right) \quad\left(x_{3}, x_{4}, x_{5}, x_{0}, x_{1}, x_{2}\right)
$$


with the six coordinates subject to (11.1). The directrix in (12.1) is the locus of

$$
\left(x_{0}+x_{3}, x_{1}+x_{4}, x_{2}+x_{5}, x_{3}+x_{0}, x_{4}+x_{1}, x_{5}+x_{2}\right)
$$

when the points (12.2) trace $\Gamma_{5}^{\prime}$. But then, by (11.1),

$$
\begin{aligned}
& x_{0}^{2}+x_{3}^{2}: x_{1}^{2}+x_{4}^{2}: x_{2}^{2}+x_{5}^{2}=b^{2}-c^{2}: c^{2}-a^{2}: a^{2}-b^{2}, \\
& x_{0}^{2}-x_{3}^{2}: x_{1}^{2}-x_{4}^{2}: x_{2}^{2}-x_{5}^{2}=b c\left(b^{2}-c^{2}\right): c a\left(c^{2}-a^{2}\right): a b\left(a^{2}-b^{2}\right)
\end{aligned}
$$

so that there are constants $\mu, v$ for which

$$
x_{0}^{2}=\left(b^{2}-c^{2}\right)(\mu+\nu b c), \quad x_{3}^{2}=\left(b^{2}-c^{2}\right)(\mu-\nu b c) .
$$

Thus the point $(\xi, \eta, \zeta, \xi, \eta, \zeta)$ on $R$ in (12.1) is such that

$$
\begin{aligned}
\xi & =\left(b^{2}-c^{2}\right)^{\frac{1}{2}}\left\{(\mu+\nu b c)^{\frac{1}{2}}+(\mu-v b c)^{\frac{1}{2}}\right\}, \\
\frac{1}{2} \xi^{2} & =\left(b^{2}-c^{2}\right)\left\{\mu+\left(\mu^{2}-v^{2} b^{2} c^{2}\right)^{\frac{1}{2}}\right\} \\
\frac{1}{4} \xi^{4} & =\left(b^{2}-c^{2}\right)^{2}\left\{2 \mu^{2}-v^{2} b^{2} e^{2}+2 \mu\left(\mu^{2}-v^{2} b^{2} c^{2}\right)^{\frac{1}{2}}\right\} \\
& =\left(b^{2}-c^{2}\right)\left\{\mu \xi^{2}-\nu^{2} b^{2} e^{2}\left(b^{2}-c^{2}\right)\right\} .
\end{aligned}
$$

Two similar relations, derived from this by simultaneous cyclic permutations $(\xi \eta \zeta)$ and $(a b c)$, also hold; elimination of $\mu$ and $\nu$ from the three relations gives

$$
\left|\begin{array}{ccc}
\xi^{4} & \left(b^{2}-c^{2}\right) \xi^{2} & b^{2} c^{2}\left(b^{2}-c^{2}\right)^{2} \\
\eta^{4} & \left(c^{2}-a^{2}\right) \eta^{2} & c^{2} a^{2}\left(c^{2}-a^{2}\right)^{2} \\
\zeta^{4} & \left(a^{2}-b^{2}\right) \zeta^{2} & a^{2} b^{2}\left(a^{2}-b^{2}\right)^{2}
\end{array}\right|=0
$$

a plane sextic with nodes, indeed biflecnodes, at the vertices of the triangle $\xi \eta \zeta=0$. Since the genus is known to be 7 three nodes on the plane curve are to be expected.

13. - The preceding paragraphs have been concerned with $\Gamma_{n}^{\prime}$ invariant, when $n=2 p+1$, under a $(p+1)$-fold transposition of the $2 p+2$ homogeneous coordinates. If one seeks by analogy a curve $\Gamma_{n}^{\prime \prime}$ invariant under a permutation, of the homogeneous coordinates, consisting wholly of 3 -cycles then $n=3 s-1$; if $\omega$ is either complex cube root of 1 take $\Gamma_{n}^{\prime \prime}$ to be defined by

$$
\Omega_{k} \equiv \sum_{j=0}^{s-1} a_{j}^{k}\left(x_{j}^{2}+\omega^{k} x_{j+s}^{2}+\omega^{2 k} x_{j+2 s}^{2}\right)=0 \quad k=0,1,2, \ldots, 3 s-3
$$

It is invariant under the projectivity $\wp$ induced by the permutation

$$
\left(x_{0} x_{s} x_{2 s}\right)\left(x_{1} x_{1+s} x_{1+2 s}\right) \ldots\left(x_{s-1} x_{2 s-1} x_{3 s-1}\right)
$$

since this merely multiplies $\Omega_{k}$ by $\omega^{2 k}$. 
The points invariant under $\wp$ are those, and only those, of the three $[s-1]$ 's

$$
\alpha: x_{j}=x_{j_{+}}=x_{j+2 s} ; \quad \beta: x_{j}=\omega x_{j_{+s}}=\omega^{2} x_{j_{+2 s}} ; \quad \bar{\beta}: x_{j}=\omega^{2} x_{j+s}=\omega x_{j_{+2 s}} ;
$$

where $j$ runs over $0,1,2, \ldots, s-1$ and each written pair of equations is but one of $s$ pairs of linear equations holding simultaneously. These three spaces are mutually skew and together span the whole $[3 s-1]$; the pairs span the $[2 s-1]$ 's,

$$
\begin{gathered}
\beta \bar{\beta}: x_{j}+x_{j_{+s}}+x_{j_{+2 s}}=0 ; \quad \alpha \bar{\beta}: x_{j}+\omega x_{j_{+s}}+\omega^{2} x_{j_{+2 s}}=0 ; \\
\alpha \beta: x_{j}+\omega^{2} x_{i_{+}}+\omega x_{j_{+2 s}}=0 .
\end{gathered}
$$

Any point not in any of $\alpha, \beta, \bar{\beta}$ is one of a trio cyclically permuted by (13.1); should the point be in any of the three $[2 s-1]$ 's the members of the trio are collinear; otherwise they span a plane meeting all of $\alpha, \beta, \bar{\beta}$. One may note the $s$ trios $X_{j} X_{j_{+} s} X_{j+2 s}$ spanned by vertices of $S$. Their $s$ intersections with $\alpha, \beta$ or $\bar{\beta}$ form a simplex in this $[s-1]$; the harmonic inversions in its vertices and opposite bounding $[s-2]$ 's being induced in $\alpha, \beta$ or $\bar{\beta}$ by

$$
h_{0} h_{s} h_{2 s}, \ldots, h_{j} h_{j+s} h_{j+2 s}, \ldots, h_{s-1} h_{2 s-1} h_{3 s-1} .
$$

These, commuting and having identity for their product, generate an elementary abelian group $e$ of order $2^{s-1}$; it is clear from (13.2) that all of them leave $\alpha, \beta, \bar{\beta}$ invariant. The three $[s-1]$ 's are thus only one of $2^{3 s-1} / 2^{s-1}=2^{2 s}$ such sets of spaces associated with $\Gamma_{n}^{\prime \prime}$, those of a set being transformed into those of any other by the $2^{\varepsilon-1}$ operations of a coset of $e$ in $E$.

$\alpha$ lies on $2 s-2$ of the $3 s-2$ quadries $\Omega_{k}=0$, namely those for which $k \neq 0$ ( $\bmod 3$ ). But each of $\beta$ and $\bar{\beta}$ lies on $2 s-1$ of the $3 s-2$ quadries : $\beta$ on those having $k \neq 2, \bar{\beta}$ on those having $k \neq 1$. It follows that $\beta$ and $\bar{\beta}$ both meet $\Gamma_{n}^{\prime \prime}$ in $2^{s-1}$ points, namely those, in the case of $\beta$, common to the sections by $\beta$ of the $s-1$ quadrics $\Omega_{k}=0$ having $k \equiv 2$ and those, in the case of $\bar{\beta}$, common to the sections by $\bar{\beta}$ of the $s-1$ quadrics $\Omega_{k}=0$ having $k \equiv 1$. The planes spanned by trios include the osculating planes of $\Gamma_{n}^{\prime \prime}$ at these $2^{s}$ points in $\beta$ and $\bar{\beta}$.

The $2^{s-1}$ points in, for example, $\beta$ satisfy

$$
\sum a_{j}^{2} x_{j}^{2}=\sum a_{j}^{5} x_{j}^{2}=\ldots=\sum a_{j}^{3 s-4} x_{j}^{2}=0 ;
$$

the obvious determinantal solution of these $s-1$ linear equations for the $s$ unknowns » $x_{j}^{2}$ shows that none of the $x_{j}$ is zero.

14. - If $\xi$ is a point on $\Gamma_{n}^{\prime \prime}$ the tangent there is the line

$$
\sum_{j=0}^{s-1} a_{j}^{k}\left(\xi_{j} x_{j}+\omega^{k} \xi_{j+s} x_{i+s}+\omega^{2 k} \xi_{j+2 s} x_{j+2 s}\right)=0 \quad k=0,1, \ldots, 3 s-3
$$


Should $\xi$ happen to be one of the points of $\Gamma_{n}^{\prime \prime}$ in $\beta$ these equations would be

$$
\sum a_{j}^{k}\left(x_{j}+\omega^{k+2} x_{j+s}+\omega^{2 k+1} x_{j+2 s}\right) \xi_{j}=0
$$

Now a point $x$ in $\alpha$ would satisfy all these equations if only, for each $k$,

$$
\sum a_{j}^{k}\left(1+\omega^{k+2}+\omega^{2 k+1}\right) x_{j} \xi_{j}=0
$$

but all these $3 s-2$ equations other than those $s-1$ for which $k \equiv 1$ are nugatory so that there are only these $s-1$ linear equations to be satisfied by the $s$ coordinates $x_{j}$, namely

$$
\sum a_{j}^{k} x_{j} \xi_{j}=0 \quad k=1,4, \ldots, 3 s-2 .
$$

Since no two $a_{j}$ are equal nor, as just remarked, is any $\xi_{j}$ zero, the matrix $\left[a_{j}^{k} \xi_{j}\right]$ has rank $s-1$ and the ratios of the $x_{j}$ are uniquely determined. So the tangents to $\Gamma_{n}^{\prime \prime}$ at its $2^{s-1}$ intersections with $\beta$ all meet $\alpha$ as, by similar reasoning, do the tangents at its $2^{s-1}$ intersections with $\bar{\beta}$.

When $\Gamma_{n}$ is specialised to $\Gamma_{n}^{\prime \prime}$ the parametric form (4.3) implies, as is seen on using (7.1) with $h=3$, the relations

$$
x_{j}^{2}: x_{j+s}^{2}: x_{j+2 s}^{2}=\theta+a_{j}: \omega\left(\theta+\omega a_{j}\right): \omega^{2}\left(\theta+\omega^{2} a_{j}\right) \quad j=0,1, \ldots, s-1 .
$$

But, on $\beta, x_{j}: x_{j_{+s}}: x_{j_{2} s}=1: \omega^{2}: \omega$ so that all $2^{s-1}$ intersections of $\Gamma_{n}^{\prime \prime}$ with $\beta$ belong to $B(\infty)$. This batch of $2^{3 s-1}$ points is completed on appropriating the points of $\Gamma_{n}^{\prime \prime}$ in those $2^{2 s}-1$ other spaces derived by applying $E$ to $\beta$. Similar remarks apply to $\bar{\beta}$ and $B(0)$.

The osculating prime of $\Gamma_{n}^{\prime \prime}$ at a point of $B(0)$ is, by (3.1) of [5] with $n=3 s-1$,

$$
\sum_{j=0}^{s-1} a_{j}^{3 s-3}\left(\xi_{j} x_{j}+\xi_{j+s} x_{j+s}+\xi_{j+2 s} x_{j+2 s}\right)=0
$$

When $\xi$ is in $\bar{\beta}$ this equation is, by (13.2).

$$
\sum a_{j}^{3 s-3}\left(x_{j}+\omega x_{j+s}+\omega^{2} x_{j+2 s}\right) \xi_{j}=0
$$

and is satisfied by every point of $\alpha \bar{\beta}$; the osculating primes of $\Gamma_{n}^{\prime \prime}$ at its $2^{s-1}$ points in $\bar{\beta}$ all contain the $[2 s-1] \alpha \bar{\beta}$. All $2^{s-2}\left(2^{s-1}-1\right)$ joins of the $2^{s-1}$ points are therefore principal chords, and the operations of $E$ applied to them produce $2^{3 s-2}\left(2^{s-1}-1\right)$ of the principal chords joining pairs of points in $B(0)$. Analogous statements hold for $B(\infty)$. 
15. - The planes of the trios generate a threefold $W$ whose genus appears on using Zeuthen's formula. For there is a $(1,3)$ correspondence between the planes of $W$ and the points of $\Gamma_{n}^{\prime \prime}$ in which the only coincidences occur at the $2^{s}$ intersections of $\Gamma_{n}^{\prime \prime}$ with $\beta$ and $\bar{\beta}$. But each of these is a coincidence of all three members of a trio and so $([8]$, p. 238$)$ is to be counted twice; whence the formula gives

$$
\begin{aligned}
6(\pi-1)+2.2^{s} & =2\left(\pi_{3 s-1}-1\right) \\
& =2.2^{3 s-3}(3 s-4),
\end{aligned}
$$

$\pi$ being the unknown genus of $W$. Thus

$$
\begin{aligned}
\pi & =1+s \cdot 2^{2 s-3}-2^{s}\left(2^{2 s-1}+1\right) / 3 \\
& =1+s \cdot 2^{3 s-3}-2^{s}\left(1-2+2^{2}-\ldots+2^{2 s-2}\right) .
\end{aligned}
$$

$\alpha, \beta, \bar{\beta}$ contain directrices of $W ; \beta \bar{\beta}, \alpha \bar{\beta}, \alpha \beta$ meet $W$ in scrolls. Among the $2^{3 s-2}$ intersections of $\Gamma_{n}^{\prime \prime}$ with a prime through $\beta \bar{\beta}$ are $2^{s-1}$ points on $\beta$ and $2^{s-1}$ on $\bar{\beta}$; the $2^{3 s-2}-2^{s}$ others consist of trios whose $M=\frac{1}{3}\left(2^{2 s-2}-1\right) 2^{s}$ planes meet $\alpha$ on the directrix there, whose order is therefore $M$. The other two directrices have the same order because a prime through $\alpha \bar{\beta}$ or $\alpha \beta$ contains the tangents at $2^{s-1}$ points of $\Gamma_{n}^{\prime \prime}$ which account for $2^{s}$ intersections. The generators of each scroll put a pair of directrices in $(1,1)$ correspondence; the scrolls have order $2 M, W$ has order $3 M$.

16. - The simple instance when $s=2$ furnishes a threefold of order 12 with three quadruple lines and merits some description. $\Gamma_{5}^{\prime \prime}$, of order 16 and genus 17 , is defined by four simultaneous equations $(a \neq b)$

$$
\begin{aligned}
x_{0}^{2}+x_{2}^{2}+x_{4}^{2}+x_{1}^{2}+x_{3}^{2}+x_{5}^{2} & =0 \\
a\left(x_{0}^{2}+\omega x_{2}^{2}+\omega^{2} x_{4}^{2}\right)+b\left(x_{1}^{2}+\omega x_{3}^{2}+\omega^{2} x_{5}^{2}\right) & =0 \\
a^{2}\left(x_{0}^{2}+\omega^{2} x_{2}^{2}+\omega x_{4}^{2}\right)+b^{2}\left(x_{1}^{2}+\omega^{2} x_{3}^{2}+\omega x_{5}^{2}\right) & =0 \\
a^{3}\left(x_{0}^{2}+x_{2}^{2}+x_{4}^{2}\right)+b^{3}\left(x_{1}^{2}+x_{3}^{2}+x_{5}^{2}\right) & =0
\end{aligned}
$$

Hence, on $\Gamma_{5}^{\prime \prime}$,

$$
\begin{gathered}
x_{0}^{2}+x_{2}^{2}+x_{4}^{2}=x_{1}^{2}+x_{3}^{2}+x_{5}^{2}=0, \\
x_{0}^{2}+\omega x_{2}^{2}+\omega^{2} x_{4}^{2}=\varrho b, \quad x_{1}^{2}+\omega x_{3}^{2}+\omega^{2} x_{5}^{2}=-\varrho a, \\
x_{0}^{2}+\omega^{2} x_{2}^{2}+\omega x_{4}^{2}=\sigma b^{2}, \quad x_{1}^{2}+\omega^{2} x_{3}^{2}+\omega x_{5}^{2}=-\sigma a^{2} ; \\
3 x_{0}^{2}=\varrho b+\sigma b^{2}, \quad 3 x_{2}^{2}=\omega^{2} \varrho b+\omega \sigma b^{2}, \quad 3 x_{4}^{2}=\omega \varrho b+\omega^{2} \sigma b^{2}, \\
-3 x_{1}^{2}=\varrho a+\sigma a^{2}, \quad-3 x_{3}^{2}=\omega^{2} \varrho a+\omega \sigma a^{2}, \quad-3 x_{5}^{2}=\omega \varrho a+\omega^{2} \sigma a^{2} .
\end{gathered}
$$


Those points that are invariant when the $x_{j}$ undergo the permutation $\left(x_{0} x_{2} x_{4}\right)\left(x_{1} x_{3} x_{5}\right)$ are on the lines

$$
\begin{array}{ll}
\alpha: x_{0}=x_{2}=x_{4}, & x_{1}=x_{3}=x_{5} \\
\beta: x_{0}=\omega x_{2}=\omega^{2} x_{4}, & x_{1}=\omega x_{3}=\omega^{2} x_{5} \\
\bar{\beta}: x_{0}=\omega^{2} x_{2}=\omega x_{4}, & x_{1}=\omega^{2} x_{3}=\omega x_{5} .
\end{array}
$$

Should a point of $\Gamma_{5}^{\prime \prime}$ be on $\beta$ then, from (16.2), $\varrho=0$; there are two intersections

$$
x_{0}^{2}: x_{2}^{2}: x_{4}^{2}: x_{1}^{2}: x_{3}^{2}: x_{5}^{2}=b^{2}: \omega b^{2}: \omega^{2} b^{2}:-a^{2}:-\omega a^{2}:-\omega^{2} a^{2} .
$$

Likewise there are two intersections with $\bar{\beta}$; but none with $\alpha$.

The solids containing collinear trios are

$$
\begin{aligned}
& \beta \bar{\beta}: x_{0}+x_{2}+x_{4}=x_{1}+x_{3}+x_{5}=0, \\
& \alpha \bar{\beta}: x_{0}+\omega x_{2}+\omega^{2} x_{4}=x_{1}+\omega x_{3}+\omega^{2} x_{5}=0, \\
& \alpha \beta: x_{0}+\omega^{2} x_{2}+\omega x_{4}=x_{1}+\omega^{2} x_{3}+\omega x_{5}=0 .
\end{aligned}
$$

Take, now, three points $\left(x_{0}, x_{2}, x_{4}, x_{1}, x_{3}, x_{5}\right)$ one on each of $\alpha, \beta, \bar{\beta}$ : say

$$
\begin{array}{ll}
(1,1,1, k, k, k) & \text { on } \alpha, \\
\left(1, \omega^{2}, \omega, m, \omega^{2} m, \omega m\right) & \text { on } \beta, \\
\left(1, \omega, \omega^{2}, n, \omega n, \omega^{2} n\right) & \text { on } \bar{\beta} .
\end{array}
$$

The object is to select $k, m, n$ so that the plane spanned by these points contains a trio on $\Gamma_{5}^{\prime \prime}$; in order that it should do so it is sufficient for it to meet $\Gamma_{5}^{\prime \prime}$ once. But every point of the plane is obtained by varying $\lambda, \mu, v$ in

$$
\begin{aligned}
& \left(\lambda+\mu+\nu, \lambda+\mu \omega^{2}+\nu \omega, \lambda+\mu \omega+\nu \omega^{2}\right. \\
& \left.\quad \lambda k+\mu m+\nu n, \lambda k+\mu \omega^{2} m+\nu \omega n, \lambda k+\mu \omega m+v \omega^{2} n\right)
\end{aligned}
$$

and on substituting these coordinates for the $x_{i}$ in (16.1) one has the conditions

$$
\begin{aligned}
\lambda^{2}+2 \mu \nu+\lambda^{2} k^{2}+2 \mu \nu m n & =0, \\
a\left(\nu^{2}+2 \lambda \mu\right)+b\left(\nu^{2} n^{2}+2 \lambda \mu k m\right) & =0, \\
a^{2}\left(\mu^{2}+2 \nu \lambda\right)+b^{2}\left(\mu^{2} m^{2}+2 \nu \lambda k n\right) & =0, \\
a^{3}\left(\lambda^{2}+2 \mu \nu\right)+b^{3}\left(\lambda^{2} k^{2}+2 \mu \nu m n\right) & =0 .
\end{aligned}
$$

17 - Annali di Matematica 
The first and fourth conditions require

$$
\lambda^{2}+2 \mu \nu=\lambda^{2} k^{2}+2 \mu \nu m n=0
$$

so that either $\lambda^{2}+2 \mu \nu=k^{2}-2 m n=0$ or $\lambda^{2}=2 \mu \nu=0$. And, in either alternative, one has to satisfy the second and third of the four conditions.

If, in the second alternative, $\lambda=\mu=0$, the third condition is identically satisfied, the second if $a+b n^{2}=0$; one obtains the two points of $\Gamma_{5}^{\prime \prime}$ on $\bar{\beta}$ and their osculating planes. Similarly, $\lambda=\nu=0$ yields the two points on $\beta$. So one considers the first alternative. The two conditions are

$$
v^{2}\left(a+b n^{2}\right)=-2 \lambda \mu(a+b k m), \quad \mu^{2}\left(a^{2}+b^{2} m^{2}\right)=-2 v \lambda\left(a^{2}+b^{2} k n\right),
$$

which give, on multiplication and cancelling $\mu \nu$,

$$
\mu v\left(a+b n^{2}\right)\left(a^{2}+b^{2} m^{2}\right)=4 \lambda^{2}(a+b k m)\left(a^{2}+b^{2} k n\right)
$$

or, since $\lambda^{2}=-2 \mu \nu$,

$$
\left(a+b n^{2}\right)\left(a^{2}+b^{2} m^{2}\right)+8(a+b k m)\left(a^{2}+b^{2} k n\right)=0 .
$$

This is the relation sought; when it is satsfied, in addition to $k^{2}=2 m n$, the plane belongs to $W$. If any one of $k, m, n$ is now eliminated the outcome is a $(4,4)$ correspondence between the other two; this yields an octavic scroll with two of $\alpha, \beta, \bar{\beta}$ for quadruple lines. For example: the elimination of $k$ leads to

$$
\left(9 a^{3}+a^{2} b n^{2}+a b^{2} m^{2}+17 b^{3} m^{2} n^{2}\right)^{2}=128 a^{2} b^{2}(a m+b n)^{2} m n .
$$

The plane (16.3) meets $\beta \bar{\beta}$ in the line

$$
\left(\mu+\nu, \mu \omega^{2}+\nu \omega, \mu \omega+\nu \omega^{2}, \mu m+\nu n, \mu \omega^{2} m+\nu \omega n, \mu \omega m+\nu \omega^{2} n\right)
$$

so that one has only to take

$$
m=\left(\omega^{2} x_{3}-\omega x_{5}\right) /\left(\omega^{2} x_{2}-\omega x_{4}\right), \quad n=\left(\omega x_{3}-\omega^{2} x_{5}\right) /\left(\omega x_{2}-\omega^{2} x_{4}\right)
$$

and substitute in (16.5) to obtain an equation for the octavic scroll $R_{\alpha}$ in which $\beta \bar{\beta}$ meets $W$.

The generators of $R_{\alpha}$ are paired in the harmonic inversion $h_{0} h_{2} h_{4}=h_{3} h_{3} h_{5}$; this accords with (16.5) being unchanged when $m, n$ are replaced by $-m,-n$. A plane section of $R_{\alpha}$ has quadruple points, one on $\beta$ and one on $\bar{\beta}$, so that, being of genus 5 by (15.1), it is required to have 4 nodes; $R_{\alpha}$ has 4 double generators. 


\section{III. - Curves invariant when the coordinates are permuted in a single cycle.}

17. - There is a specialisation of $\Gamma_{n}$ having no surviving moduli; the properties of such a curve $\Delta_{n}$ include those of the less specialised curves already considered.

Take $a_{j}=\varepsilon^{j}$ where $\varepsilon=\exp [2 \pi i /(n+1)]$, a primitive $(n+1)$-th root of unity. Then $\Delta_{n}$ admits, in addition to $E$, the cyclic self-projectivity $\wp$, of period $n+1$, that permutes the coordinates in a single cycle $\left(x_{0} x_{1} \ldots x_{n}\right)$. For this permutation merely divides $\Omega_{k}$ by $\varepsilon^{k}$ because, now,

$$
\Omega_{k} \equiv \sum \varepsilon^{j k} x_{j}^{2}
$$

Every quadric through $\Delta_{n}$ is invariant under a group of $2^{n}(n+1)$ projectivities; though $\Delta_{n}$ itself will be invariant for a larger group should there be projectivities permuting the quadrics through $\Delta_{n}$ among themselves.

Since, now,

$$
f(\theta)=\theta^{n+1}-1, \quad f^{\prime}(\theta)=(n+1) \theta^{n}, \quad f^{\prime}\left(\varepsilon^{j}\right)=(n+1) \varepsilon^{-j}
$$

the parametrisation $(4.3)$ is

$$
x_{i}^{2}=\left(\theta+\varepsilon^{j}\right) \varepsilon^{j} .
$$

When $\wp$ is imposed $B(\theta)$ becomes $B(\varphi)$ where the ratio

$$
\left(\theta+\varepsilon^{j}\right) \varepsilon^{j}:\left(\varphi+\varepsilon^{j+1}\right) \varepsilon^{j+1}
$$

is independent of $j$, hence $\varphi=\varepsilon \theta$. The batches are permuted in cycles of $n+1$ save that $B(0)$ and $B(\infty)$ are unmoved. The $n+1$ critical batches are $B\left(-\varepsilon^{j}\right)$.

18. $-\wp$ has the $n+1$ distinct fixed points

$$
A_{k}: x_{j}=\varepsilon^{j k} \quad k=0,1, \ldots, n,
$$

vertices of a simplex $\subseteq$. Each $A_{k}$ lies on all $n+1$ quadrics $\Omega_{k}$ except $\Omega_{n+1-2 k}$ (the suffix modulo $n+1$ ) so that $A_{k}$ is on $A_{n}$ when $\Omega_{n+1-2 k}$ is either $\Omega_{n-1}$ or $\Omega_{n}$. Thus $A_{1}$, not being on $\Omega_{n-1}$, is on $\Delta_{n}$, indeed in $B(0)$.

If $n=2 p+1$ is odd, so that $\varepsilon^{p+1}=-1$, the inversion (18.1) in a pair of opposite bounding [ $p$ ]'s of $S$ transposes $A_{1}$ and $A_{p_{+2}}$ so that this last point is also on $A_{n}$ and in $B(0)$.

If $n=2 q$ is even, $A_{q_{+1}}$ is not on $\Omega_{n}$ and so is on $\Delta_{n}$; indeed it belongs to $B(\infty)$. 
Consider now the osculating spaces of $A_{n}$ at $A_{1}$. Since, at $A_{1}, \theta=0$ and $\xi_{j}=\varepsilon^{i}$ the osculating [s] is, as explained in [5], determined by the $n-s$ linear equations

$$
\sum\left(\varepsilon^{j}\right)^{r-2} \varepsilon^{j} x_{j}=0 \quad \text { or } \quad \sum \varepsilon^{(r-1) i} x_{j}=0 \quad r=n, n-1, \ldots, s+1 .
$$

But this is the equation of that bounding prime of $\subseteq$ that is opposite to $A_{n-q+2}$ and so one can say, in suecession, that

$$
\begin{aligned}
& \text { the osculating }[n-1] \text { at } A_{1} \text { is } A_{3} A_{4} A_{5} \ldots A_{n} A_{0} A_{1}, \\
& \text { the osculating }[n-2] \text { at } A_{1} \text { is } A_{4} A_{5} \ldots A_{n} A_{0} A_{1} \text {, } \\
& \ldots \ldots \ldots \\
& \text { the osculating plane at } A_{1} \text { is } \\
& \text { the tangent at } A_{1} \text { is } \\
& \qquad A_{n} A_{0} A_{1} \text {, } \\
& A_{0} A_{1} .
\end{aligned}
$$

Or, building the figure from the spaces of lower dimension, the tangent of $\Delta_{n}$ at $A_{1}$ is $A_{1} A_{0}$, the osculating plane $A_{1} A_{0} A_{n}$, the osculating solid $A_{1} A_{0} A_{n} A_{n-1}$, and so on.

Suppose now that $n=2 p+1$; the alternative $n=2 q$ will be discussed below.

The inversion

$$
h_{0} h_{2} \ldots h_{2 p} \equiv h_{1} h_{2} \ldots h_{2 p+1}
$$

replaces the coordinate $\varepsilon^{j k}$ of $A_{k}$ by $(-1)^{j} \varepsilon^{j k}$ or $\varepsilon^{j(k+p+1)}$, and so transposes $A_{k}$ and $A_{k+y+1}$ (suffixes modulo $\left.2 p+2\right)$; the vertices of $\subseteq$ undergo the $(p+1)$-fold transposition

$$
\left(A_{0} A_{p+1}\right)\left(A_{1} A_{p+2}\right) \ldots\left(A_{p} A_{2 p+1}\right)
$$

Hence the tangent of $A_{2 p+1}$ at $A_{p+2}$ is $A_{p+2} A_{p+1}$, the osculating plane $A_{p+2} A_{p+1} A_{p}$, and so on, the osculating $[2 p]$ being the bounding prime

$$
A_{p+2} A_{p+1} \ldots A_{2 p} A_{0} \ldots A_{p+4}
$$

of $\subseteq$ opposite $A_{p+3}$.

Clearly $A_{1} A_{\mathfrak{p + 2}}$ is a principal chord; but there is more to say. For $A_{1} A_{p+2}$ is common to

$$
A_{1} A_{0} A_{2 p+1} A_{2 p} \ldots A_{p+2} \quad \text { and } \quad A_{p+2} A_{p+1} \ldots A_{2} A_{1}
$$

which are the osculating $[p+1]$ 's at $A_{1}$ and $A_{y_{+2}}$ respectively. Take, for example, $p=2$, Then the chord $A_{1} A_{4}$ of $A_{5}$ lies not only in the osculating [4]'s at both $A_{1}$ and $A_{4}$ but also in the osculating solids

$$
A_{1} A_{0} A_{5} A_{4} \quad \text { and } \quad A_{4} A_{3} A_{2} A_{1}
$$


$A_{1} A_{4}$ is one of 16 such ehords joining pairs in the batch $B(0)$; the members of a pair are images of one another in the inversion $h_{0} h_{2} h_{4}$.

It was seen, in the account of $\Gamma_{5}^{\prime}$, that any point $P$ in $B(0)$ is joined to three other points $P_{03}, P_{14}, P_{25}$ of the batch by principal chords, and that every edge of the tetrahedron $P P_{03} P_{14} P_{25}$ is such a chord. The four points are transforms of any one by $h_{0} h_{3}, h_{1} h_{4}, h_{2} h_{5} ;$ e.g.

$$
h_{2} h_{5} P_{03}=h_{2} h_{5} h_{0} h_{3} P=h_{1} h_{4} P=P_{14} .
$$

This is therefore true also of $\Delta_{5}$, since invariance under the involution $\wp^{3}$ endows $\Delta_{5}$ with the properties of $\Gamma_{5}^{\prime}$. But invariance under $\wp^{2}$ endows $\Delta_{5}$ with the properties of $\Gamma_{5}^{\prime \prime}$ also; among them is the fact that there is a fourth principal chord joining $P$ to another point $P_{024}$ of $B(0)$, the transform of $P$ by $h_{0} h_{2} h_{4}$. It is this last chord which, as has just been noted, now lies not only in the osculating [4]'s but also in the osculating solids of $A_{5}$ at both $P$ and $P_{024}$. The joins of pairs of points in $B(0)$ include $48+16=64$ principal chords of $\Delta_{5}$.

19. - Suppose now, still considering $A_{n}$, that $n$ is even, say $n=2 q$. The projectivity

$$
J: x_{j}=\varepsilon^{(q+2) i} x_{2 q+1-j} \quad j=1,2, \ldots, 2 q
$$

with the first coordinate $x_{0}$ unchanged, is seen to transpose $\Omega_{k}$ and $\Omega_{k^{\prime}}$ when $k+k^{l}=2 q-2$. In detail

$$
\varepsilon^{i k} x_{j}^{2} \quad \text { becomes } \varepsilon^{j(k+2 q+4)} x_{2 q+1-j}^{2}
$$

so that the typical term of the sum into which $\Omega_{k}$ is transformed is, changing $j$ to $2 q+1-j$

$$
\varepsilon^{(2 q+1-j)(k+2 \alpha+4)} x_{j}^{2}=\varepsilon^{-j(k+3)} x_{j}^{2}=\varepsilon^{j(2 q-k-2)} x_{j}^{2}
$$

since $\varepsilon^{2 q+1}=1$. Thus $J$ transposes the $n-1$ quadrics through $\Delta_{2 q}$ in pairs, save that $\Omega_{q-1}$ is invariant. $J$ also, as can be similarly verified, transposes $\Omega_{n-1}$ and $\Omega_{n}$.

A matrix form for $J$ is

$$
J=\left[\begin{array}{cccc} 
\pm 1 & \cdot & \cdot & \cdot \\
\cdot & \cdot & \cdot & \varepsilon^{a+2} \\
\cdot & \cdot & \varepsilon^{2 \alpha+4} & \cdot \\
\cdot & \varepsilon^{2 q(q+2)} & \cdot & \cdot
\end{array}\right]
$$

(the sign of the leading element being chosen so that $|J|=+1$ ) having the unit 
matrix for its square; $J$ is an involution. If $P$ is the $(2 q+1)$-rowed permutation matrix

$$
\left[\begin{array}{cccc}
\cdot & 1 & \cdot & \cdot \\
\cdot & \cdot & 1 & \cdot \\
\cdot & \cdot & \cdot & 1 \\
1 & \cdot & \cdot & \cdot
\end{array}\right]
$$

imposing $\wp$ it appears, since $J P J=\varepsilon^{q+2} P^{-1}$ that $J$ and $\wp$ generate a dihedral group of $4 q+2$ projectivities. The $4 q+2$ matrix forms for these can, on multiplication by the diagonal matrices imposing projectivities of $E$, have the signs of any of their entries changed and yet still impose projectivities that leave $\Delta_{2 q}$ invariant; the group of self-projectivities has order $(4 q+2) 2^{2 q}$ and can be imposed by as many matrices of determinant +1 (should $q$ be odd write the leading entry of $y$ as -1 ).

This order is 160 when $q=2$. So far as is known $\Delta_{n}$ has never been encountered save in this simplest instance, but $\Delta_{4}$ was discovered by Wiman in his quest [11] for curves of low genus admitting self-projectivities. He did not, however, exploit his discovery other than by giving this order 160. Some further contributions to the study of $\Delta_{4}$ have since appeared in [3]; a little more appears below.

The characteristic polynomial of $\mathfrak{J}$ is, if the leading element is +1 ,

$$
(1-\lambda)\left(\lambda^{2}-1\right)^{q}
$$

and there is no obstacle to perceiving that, corresponding to the latent root +1 , $q+1$ linearly independent latent vectors span the $[q]$

$$
Q: x_{r+1}=\varepsilon^{(q+2)(r+1)} x_{2 q-r}
$$

while, corresponding to the latent root $-1, q$ linearly independent latent vectors span the $[q-1]$

$$
Q^{\prime}: x_{0}=x_{r+1}+\varepsilon^{(q+2)(r+1)} x_{2 q-r}=0
$$

Here $r$ ranges over $0,1, \ldots, q-1$. Both $Q$ and $Q^{\prime}$ meet all of

$$
X_{1} X_{2 q}, X_{2} X_{2 q-1}, \ldots, X_{\alpha} X_{q+1}
$$

$Q$ also contains $X_{0}$, while $Q^{\prime}$ lies in $x_{0}=0$.

$J$ is the harmonic inversion in $Q$ and $Q^{\prime}$ which, $\Omega_{q_{-1}}$ being invariant, are polar spaces for $\Omega_{q-1}=0$. Furthermore: since $\Omega_{k}$ and $\Omega_{k^{\prime}}$ are transposed when $k+k^{\prime}=$ $=2 q-2$ any point in which either quadric meets either $Q$ or $Q^{\prime}$ is on the other quadric. 
Hence $\Delta_{2 q}$ meets $Q$ in the $2^{a}$ points

$$
\Omega_{0}=\Omega_{1}=\ldots=\Omega_{\alpha-1}=0
$$

these quadrics have no other common point in $Q$. Moreover the $2^{q}$ tangents of $\Delta_{2 q}$ at these points, being invariant under $J$, all meet $Q^{\prime}$; the points themselves are paired, the $2^{a-1}$ joins of the pairs all passing through $X_{0}$.

$Q$ and $Q^{\prime}$ are but one of $(4 q+2) 2^{q-1}$ pairs of polar spaces for $\Omega_{q-1}=0$ that are axes of involutions leaving $\Delta_{2 q}$ invariant. For $Q, Q^{\prime}$ are themselves invariant under

$$
h_{0}, h_{1} h_{2 q}, h_{2} h_{2 q-1}, \ldots, h_{q} h_{q+1}, J
$$

The first $q+1$ of these $q+2$ involutions generate an elementary abelian group of order $2^{q}$ which is amplified by $J$ to a non-abelian group of order $2^{a+1}$ whose projectivities are imposed by unimodular matrices either diagonal or having the shape of $J$. So there are as many polar pairs $Q, Q^{\prime}$ as there are cosets of a group of order $2^{q+1}$ in one of order $(4 q+2) 2^{2 q}$. Each vertex $X_{j}$ of $S$ is in $2^{a}$ of the spaces $Q$; the polars $Q^{\prime}$ of these lie in $x_{j}=0$.

20. - The points of $\Delta_{2 q}$ are paired by $J$; the chords, all transversal to $Q$ and $Q^{\prime}$, joining these pairs generate a scroll $R_{0}$ which meets $Q$ in a directrix curve $\delta_{0}$, of order $m$ say, with a point of multiplicity $2^{q-1}$ at $X_{0} . R_{0}$ has a double curve $\varrho_{0}$, of order $\mu$ say, in $Q^{\prime}$ since each generator of $R_{0}$ meets $Q^{\prime}$-which lies in $x_{0}=0$-in the same point as does its transform by $h_{0}$ (under which $R_{0}$ is invariant).

A prime through $Q^{\prime}$ contains those $m$ generators of $R_{0}$ that pass through its intersections with $\delta_{0}$, so that it meets $\Delta_{2 q}$ in $2 m$ points; whence $m=2^{2 q-2}$. But the $2^{2 q-1}$ intersections of $\Delta_{2 q}$ with a prime through $Q$ consist of the $2^{\alpha}$ points in $Q$ itself and two points on each of the $2 \mu$ generators of $R_{0}$ that pass through the $\mu$ intersections of the prime with $\varrho_{0}$; whence

$$
2^{2 q-1}=2^{q}+4 \mu, \quad \mu=2^{2 q-3}-2^{q-2} .
$$

Since the section of $R_{0}$ by this prime consists of $\delta_{0}$ and the $2 \mu$ generators the order of $R_{0}$ is

$$
m+2 \mu=2^{q-1}\left(2^{\alpha}-1\right) .
$$

A $[q-1]$ through $X_{0}$ and lying in $Q$, unless its position is specialised, meets $\delta_{0}$ in $2^{2 q-2}-2^{q-1}$ further points collinear with $X_{0}$ in pairs. Any contacts of the $[q-1]$ with branches of $\delta_{0}$ at $X_{0}$ must involve further intersections to an even number; one expects therefore all the branches to be inflectional.

The genus $\pi_{\alpha}^{\prime}$ of $R_{0}$ is found immediately from Zeuthen's formula. For $\delta_{0}$ is in $(1,2)$ correspondence with $A_{2 q}$, the branch points being the $2^{a}$ points where gene- 
rators of $R_{0}$ are tangents of $A_{2 q}$. Hence, using the known value of $\pi_{2 q}$ on the right,

$$
\begin{aligned}
4\left(\pi_{q}^{\prime}-1\right)+2^{q} & =2 \cdot 2^{2 \alpha-2}(2 q-3), \\
\pi_{q}^{\prime} & =2^{2 \alpha-3}(2 q-3)-\left(2^{q-2}-1\right) .
\end{aligned}
$$

One can also find the genus $\pi_{\alpha}^{\prime \prime}$ of $\varrho_{0}$. Any chord $P P_{0}$ of $\Delta_{2 q}$ through $X_{0}$ is paired by $J$ with a second such chord $P^{\prime} P_{0}^{\prime} ; P P^{\prime}$ and $P_{0} P_{0}^{\prime}$ concur on $\varrho_{0}$ and meet $x_{0}=0$ on the doubly covered $\Delta_{2 q-1}$ that is the projection of $\Delta_{2 \alpha}$ from $X_{0}$. Hence there is a $(1,2)$ correspondence between $\varrho_{0}$ and $\Delta_{2 q_{-1}}$ and so, the genus of $\Delta_{2 q_{-1}}$ being known, it only remains to find where the correspondence branches. There are two types of point on $\varrho_{0}$ where it does so.

(a) If $P$ is one of the $2^{a}$ intersections of $A_{2 q}$ with $Q$ it coincides with $P^{\prime}$; only one point of $\Delta_{2 q-1}$ arises, namely that on $X_{0} P P_{0} \equiv X_{0} P^{\prime} P_{0}^{\prime}$. The tangents of $A_{2 q}$ at $P$ and $P_{0}$ meet at a branch point on $\varrho_{0}$; there are $2^{a-1}$ of these.

(b) The $2^{q-1}$ generators $P P^{\prime}$ of $R_{0}$ through $X_{0}$ each give rise to coincidences $P^{\prime} \equiv P_{0}, P \equiv P_{0}^{\prime}$; such a generator meets $\varrho_{0}$ in a branch point, the only point of $\triangle_{2 q-1}$ corresponding to it being on $X_{0} P P^{\prime} \equiv X_{0} P_{0}^{\prime} P_{0}$. There are $2^{q-1}$ branch points of this type also.

The Zeuthen formula therefore gives

$$
\begin{aligned}
4\left(\pi_{q}^{\prime \prime}-1\right)+2^{q-1}+2^{q-1} & =2 \cdot 2^{2 q-3}(2 q-4) \\
\pi_{q}^{\prime \prime} & =2^{2 q-3}(q-2)-\left(2^{q-2}-1\right) .
\end{aligned}
$$

21. $-A_{2 Q}$ is invariant under $J$ which transforms $A_{k}$, having its $(j+1)$-th coordinate $\varepsilon^{j k}$ into the point for which

$$
x_{j}=\varepsilon^{(\alpha+2) j+(2 \alpha+1-j) k}=\varepsilon^{(\alpha+2-k) j}
$$

since $\varepsilon^{2 q+1}=1$; so $J$ transposes $A_{k}$ and $A_{k^{\prime}}$ where $k+k^{\prime} \equiv q+2(\bmod 2 q+1)$. Since $A_{1}$ is on $A_{2 q}$ so, as already seen otherwise in $\S 18$, is $A_{q+1} ; J$ transposes not only $A_{1}$ and $A_{a+1}$ themselves but also

the tangents $A_{1} A_{0}$ and $A_{q+1} A_{q+2}$

the osculating planes $A_{1} A_{0} A_{2 \alpha}$ and $A_{q+1} A_{q+2} A_{q+3}$,

and finally the osculating primes, these being the bounding primes of $\subseteq$ opposite $A_{2}$ and $A_{q}$. 
It now appears that

$$
A_{1} A_{0} A_{2 q} \ldots A_{q+2} A_{q+1}
$$

is the osculating $[q+1]$ of $A_{2 q}$ at both $A_{1}$ and $A_{q+1}$. This $[q+1]$ is fixed under both $\wp$ and $J$, but it is one of $2^{2 q}$ such biosculating spaces obtainable from any one of them by using $E$; the two contacts are one in each of $B(0)$ and $B(\infty)$, and the wholes of those two batches are thereby accounted for.

22. - For Wiman's curve $\Delta_{4} q=2$ and $\varepsilon^{5}=1, \Delta_{4}$ being defined ([11], p. 39 as modified in [3], p. 1265) by

$$
\sum x_{j}^{2}=\sum \varepsilon^{j} x_{j}^{2}=\sum \varepsilon^{2 j} x_{j}^{2}=0
$$

with summations running over $j=0,1,2,3,4$. This curve lies on 20 sextic scrolls of genus 2, each of them having a directrix plane quartic and a nodal line which is the polar line of the plane of the quartic in $\sum \varepsilon^{j} x_{j}^{2}=0$. Each solid $x_{j}=0$ contains four of these lines; they form a skew quadrilateral each pair of whose opposite sides is a pair of polar lines for $\sum \varepsilon^{j} x_{j}^{2}=0$; for example, the lines in $x_{0}=0$ are

$$
\begin{aligned}
& x_{1}+\varepsilon^{4} x_{4}=x_{2}+\varepsilon^{3} x_{3}=0, \quad x_{1}-\varepsilon^{4} x_{4}=x_{2}-\varepsilon^{3} x_{3}=0, \\
& x_{1}-\varepsilon^{4} x_{4}=x_{2}+\varepsilon^{3} x_{3}=0, \quad x_{1}+\varepsilon^{4} x_{4}=x_{2}-\varepsilon^{3} x_{3}=0 .
\end{aligned}
$$

Consider now the scroll $R_{0}$ with nodal line

$$
Q^{\prime}: x_{0}=x_{1}+\varepsilon^{4} x_{4}=x_{2}+\varepsilon^{3} x_{3}=0 .
$$

The polar plane of $Q^{\prime}$ in $\Omega_{1}=0$ is

$$
Q: x_{1}-\varepsilon^{4} x_{4}=x_{2}-\varepsilon^{3} x_{3}=0
$$

and meets $\Omega_{0}=0$ and $\Omega_{2}=0$ in the same conic; indeed the plane meets $A_{4}$ in those four points for which

$$
x_{0}^{2}+\left(1+\varepsilon^{3}\right) x_{4}^{2}+(1+\varepsilon) x_{3}^{2}=x_{0}^{2}+2 \varepsilon^{4} x_{4}^{2}+2 \varepsilon^{3} x_{3}^{2}=0 .
$$

The tangents to $\Delta_{4}$ at these points all meet $Q^{\prime}$. $R_{0}$ has a node at $X_{0}$ and meets $Q$ in a quartic curve $\delta_{0}$ with a biflecnode at $X_{0}$, each branch having an inflection there.

Since the difference of the two vectors

$$
\begin{aligned}
& \left(x_{0}, \quad x_{1}, \quad x_{2}, \quad x_{3}, \quad x_{4}\right) \\
& \left(x_{0}, \quad \varepsilon^{4} x_{4}, \quad \varepsilon^{3} x_{3}, \quad \varepsilon^{2} x_{2}, \quad \varepsilon x_{1}\right)
\end{aligned}
$$


registers a point on $Q^{\prime}$ their sum registers a point in $Q ; \delta_{0}$ is the locus of the point

$$
\left(X, Y, Z, \varepsilon^{2} Z, \varepsilon Y\right) \quad \text { with } \quad X: Y: Z=2 x_{0}: x_{1}+\varepsilon^{4} x_{4}: x_{2}+\varepsilon^{3} x_{3}
$$

as $\left(x_{0}, x_{1}, x_{2}, x_{3}, x_{4}\right)$ varies on $\Delta_{4}$. An equation for $\delta_{0}$ is obtained by eliminating the five $x_{j}$ from (22.1) and (22.2). As expeditious a way as any is probably to note that

$$
\left(\varepsilon+1+\varepsilon^{4}\right) x_{1}^{2} x_{4}^{2}+\left(\varepsilon^{3}+1+\varepsilon^{2}\right) x_{2}^{2} x_{3}^{2}=x_{0}^{4}
$$

everywhere on $\Delta_{4}$, for this is seen to hold identieally in $\theta$ on using (with $n=4$ ) the parametrization (17.1). The outcome is

$$
\varepsilon^{2}\left(\varepsilon+1+\varepsilon^{4}\right) Y^{4}+\varepsilon^{4}\left(\varepsilon^{3}+1+\varepsilon^{2}\right) Z^{4}=\frac{1}{2} X^{2}\left\{\varepsilon\left(\varepsilon^{3}-1+\varepsilon^{2}\right) Y^{2}+\varepsilon^{2}\left(\varepsilon-1+\varepsilon^{4}\right) Z^{2}\right\}
$$

a plane quartic with a biflecnode at $Y=Z=0$.

23. - The fact of the 16 principal chords joining points in $B(0)$ to points in $B(\infty)$ lying in biosculating solids is an as yet unremarked property of $\Delta_{4}$; although the fact that a special form of Humbert's plane sextic was a projection of $\Delta_{4}$ from a chord was noted and exploited in [3] the chord was not, apart from the possibility of its being a tangent, specially selected. Were it, however, to be one of these 16 principal chords the projection $H$ would have two inflections $I$ and $I^{\prime}$ with the same tangent, this tangent being the intersection of the plane $\tilde{w}$ of $H$ with the biosculating solid.

Project $\Delta_{4}$ from its chord $A_{1} A_{3}$ onto the opposite plane $\tilde{w} \equiv A_{0} A_{2} A_{4}$ of $\mathfrak{\Im}$; the biosculating solid, opposite $A_{2}$, is $\sigma_{3}=0$ where

so that

$$
\sigma_{k} \equiv \sum_{j=0}^{4} \varepsilon^{j k} x_{j} \quad k=0,1,2,3,4
$$

and

$$
5 x_{j} \equiv \sum_{k=0}^{4} \varepsilon^{-i k} \sigma_{k} \quad j=0,1,2,3,4
$$

$$
\left\{\begin{array}{l}
5 \Omega_{0} \equiv \sigma_{0}^{2}+2 \sigma_{2} \sigma_{3}+2 \sigma_{1} \sigma_{4} \\
5 \Omega_{1} \equiv \sigma_{3}^{2}+2 \sigma_{2} \sigma_{4}+2 \sigma_{0} \sigma_{1} \\
5 \Omega_{2} \equiv \sigma_{1}^{2}+2 \sigma_{0} \sigma_{2}+2 \sigma_{3} \sigma_{4}
\end{array}\right.
$$

In order to obtain the equation of $H$, the projection of $\Delta_{4}$ from $\sigma_{0}=\sigma_{3}=\sigma_{1}=0$, one has only to eliminate $\sigma_{2}$ and $\sigma_{4}$ from the equations got by equating the three quadratic forms simultaneously to zero. The first and third equations can be solved 
for $\sigma_{2}$ and $\sigma_{4}$ and the solution substituted in the second. The outcome is, writing $X$, $Y, Z$ for $\sigma_{0}, \sigma_{1}, \sigma_{3}$,

$$
2 Z^{6}-5 X^{2} Y^{2} Z^{2}-\left(X^{5}+X^{5}\right) Z+5 X^{3} Y^{3}=0,
$$

showing clearly that $Z=0$ is a bitangent with both contacts inflections.

The action of $\wp$ multiplies $\sigma_{k}$ by $\varepsilon^{-k}$, inducing the replacement of $X, Y, Z$ by $X$, $\varepsilon^{-1} Y, \varepsilon^{-3} Z$; this accords with $H$ being unchanged under the projectivity of period 5 which replace $X, Y, Z$ by $\varepsilon^{3} X, \varepsilon^{2} Y, Z$. Moreover, since $J$ permutes the $\sigma_{k}$ as $\left(\sigma_{0} \sigma_{1}\right)\left(\sigma_{2} \sigma_{4}\right)\left(\sigma_{3}\right)$, it induces in $\tilde{w}$ the harmonic inversion which transposes $X$ and $Y$ while leaving $Z$ unchanged. Thus $B$ is invariant under a dihedral group $D$ of 10 selfprojectivities.

24. - The general properties of Humbert's sextic are of course possessed by this specialised form $H$, indeed are enhanced by the presence of $D$. This is not the place to elaborate them, but one may remark in passing that $A_{1} A_{3}$ is a line on $F$, the cyclide $\Omega_{0}=\Omega_{2}=0$, and that, the tangent plane of $\not F$ at $x_{j}=\lambda \varepsilon^{j}+\mu \varepsilon^{3 j}$ on $A_{1} A_{3}$ being

$$
\lambda \sigma_{1}+\mu \sigma_{3}=\lambda \sigma_{3}+\mu \sigma_{0}=0,
$$

the tangent planes to $F$ at the points of $A_{1} A_{3}$ generate the line-cone $\sigma_{0} \sigma_{1}=\sigma_{3}^{2}$. Hence (see $\$ 9$ of [2]) the conic through the five nodes of $H$ is $X Y=Z^{2}$, and the two tangents at any node of $H$ pass one through $I$ and one through $I^{\prime}$.

The operations of $E$ all leave $F$, as well as its intersection $\Delta_{4}$ with $\Omega_{1}=0$, invariant, and $F$ is projected onto $\widetilde{w}$ from $A_{1} A_{3}$ by a transformation $(1,1)$ save for five "exceptional " lines on $F$ that meet $A_{1} A_{3}$. So the operations of $E$ induce Cremona transformations in $\tilde{w}$ that leave $H$ invariant: $H$ admits a group of 160 Cremona self-transformations.

The sections of $F$ by solids are projected from $A_{1} A_{3}$ into the adjoint cubics of $H$. Now while a general canonical eurve of genus 5 has 496 quadritangent solids -this being the number $2^{p-1}\left(2^{p}-1\right)$ when $p=5$-it was remarked in [2], $\$ 17$, that $A_{4}$, indeed that $\Gamma_{4}$, has ten singly-infinite families: if $t$ is a tangent the solid joining $t$ to the edge $X_{j} X_{k}$ of $S$ also contains the tangents $t_{j}, t_{k}, t_{j}$ obtained by applying $h_{j}$ and $h_{k}$ to $t . A_{4}$, however, is now seen to have 16 bioseulating solids quite separate from these ten families of quadritangent solids; $\sigma_{3}=0$ does not contain any edge, nor indeed any vertex, of $\$$. Those 15 of the 16 that do not contain $A_{1} A_{3}$ meet $F$ in elliptic quartics whose projections onto $\widetilde{w}$ are adjoint cubies of $H$ each having two 4-point intersections with it (and containing its five nodes).

25. - The involutions in $D$ form the coset of its cyclic subgroup and all transpose $I$ and $I^{\prime}$; the projectivities of the cyclic subgroup leave both $I$ and $I^{\prime}$ unmoved so 
that if $I$ and $I^{\prime}$ are designated as Poncelet's "circular " points in an extended Euclidean plane this cyclic subgroup will consist of rotations.

If, then, one puts

$$
Z=1, \quad X=\mathfrak{X}+i \mathfrak{Y}, \quad Y=\mathfrak{X}-i \mathfrak{Y}
$$

in (23.2) one obtains

$$
5\left(\mathfrak{X}^{2}+\mathfrak{Y}^{2}\right)^{2}\left(\mathfrak{X}^{2}+\mathfrak{Y}^{2}-1\right)+2=2 \mathfrak{X}\left(\mathfrak{X}^{4}-10 \mathfrak{X}^{2} \mathfrak{Y}^{2}+5 \mathfrak{Y}^{4}\right)
$$

and the right-hand side here is

$$
32 \prod_{j=0}^{4}\left(\mathfrak{X} \cos \frac{2 j \pi}{5}-\mathfrak{Y} \sin \frac{2 j \pi}{5}\right)
$$

Now put $\mathfrak{X}=\mathfrak{R} \cos \Theta, \mathfrak{Y}=\mathfrak{R} \sin \Theta$ to obtain

$$
5 \mathfrak{R}^{4}\left(\mathcal{R}^{2}-1\right)+2=32 \mathcal{R}^{5} \prod_{j=0}^{4} \cos \left(\Theta+\frac{2 j \pi}{5}\right)=2 \mathcal{R}^{5} \cos 5 \Theta .
$$

The invariance under rotation is obvious, as it is under reflection in $\Theta=0$. One is, however, denied the pleasure of a visual representation: the odd function $5\left(R-R^{-1}\right)+2 R^{-5}$ has a single minimum value 2 (when $R=1$ ) and otherwise, for $R>0$, exceeds 2 , so that $H$ has no real points save its five nodes-acnodes according to Cayley's nomenclature.

Each involution of $D$ is a central harmonic inversion with centre on $I I^{\prime}$; its axis joins the harmonic conjugate, with respect to $I$ and $I^{\prime}$, of this centre to one of the five nodes of $H$, the other four nodes being transposed in pairs.

Although $H$ has no real points other than its nodes this need not inhibit one from saying that every circle $X Y=k Z^{2}$ cuts $H$ in a decad of points invariant under D. This decad may, for certain values of $k$, be a repeated pentad at each point of which the circle touches $H$; it is just that the points will have complex coordinates. Each such pentad, invariant with $H$ and the circle, must have one vertex on an axis of each of the five involutions. Hence there will be four such " contact circles " because an axis meets $H$ in four points apart from the node through which it passes.

This accords with the Jacobian set of the $g_{10}^{1}$ cut, apart from $I$ and $I^{\prime}$, on $H$ by the circles having $([8], \S 33) 20+10-2=28$ points. Of these, 20 are on the four contact circles, and the defect is made up if each of $I$ and $I^{\prime}$ accounts for four members of the Jacobian set. And this they do because the line $I I^{\prime}$ repeated is a member of the pencil of circles, so that one of the decads consists of $I$ and $I^{\prime}$ both taken five times. Although the assessment of multiplicity can be a subtle problem this present instance is of the "elementary "type whose solution is given in $\S 36$ of [8], and other places. 
26. - It is also worth recording that the osculating planes of $\Delta_{4}$ at $A_{1}$ and $A_{3}$, lying as they do in a biosculating solid, have a line in common: indeed $A_{0} A_{4}$. The projection of $A_{4}$ from $A_{0} A_{4}$ onto the opposite plane $A_{1} A_{3} A_{2}$ of $\subseteq$ is a plane octavic $\mathrm{C}$ with triple points at $A_{1}$ and $A_{3}$. But there is only a single tangent at each triple point, and this is the same- $A_{1} A_{3}$ itself-at both. The equation of $\mathrm{C}$ is the result of eliminating, now, $\sigma_{0}$ and $\sigma_{1}$ from (23.1). The elimination of $\sigma_{0}$ from the second and third equations gives

$$
\sigma_{1}^{3}+2 \sigma_{1} \sigma_{3} \sigma_{4}-\sigma_{2}\left(\sigma_{3}^{2}+2 \sigma_{2} \sigma_{4}\right)=0
$$

while its elimination from the first and third gives

$$
\left(\sigma_{1}^{2}+2 \sigma_{3} \sigma_{4}\right)^{2}+8 \sigma_{2}^{2}\left(\sigma_{2} \sigma_{3}+\sigma_{1} \sigma \sigma_{4}\right)=0
$$

Combinations of these two results furnish the two quadratic equations for $\sigma_{1}$ :

$$
\begin{aligned}
& \sigma_{1}^{2}\left(\sigma_{3}^{2}+10 \sigma_{2} \sigma_{4}\right)+8 \sigma_{1} \sigma_{2}^{2} \sigma_{3}+2 \sigma_{3} \sigma_{4}\left(\sigma_{3}^{2}+2 \sigma_{2} \sigma_{4}\right)=0 \\
& 2 \sigma_{1}^{2} \sigma_{3} \sigma_{4}+\sigma_{1} \sigma_{2}\left(\sigma_{3}^{2}+10 \sigma_{2} \sigma_{4}\right)+4 \sigma_{3}\left(\sigma_{3} \sigma_{4}^{2}+2 \sigma_{2}^{3}\right)=0 .
\end{aligned}
$$

Elimination of $\sigma_{1}$ between them yields the sought equation for $\mathrm{C}$ namely, replacing $\sigma_{2}$, $\sigma_{3}, \sigma_{4}$ by $X, Y, Z$,

$$
Z^{8}+360 Z^{4} X^{3} Y^{2}+512 Z^{3}\left(X^{5}+Y^{5}\right)+2240 Z^{2} X^{3} Y^{3}+2000 X^{4} Y^{4}=0 .
$$

The triple points $A_{1}, A_{3}$, with their single tangent along $Z=0$, are manifest, as is invariance under $\mathfrak{D}$.

The pencil of conics $X Y=7 Z^{2}$ cut, apart from $A_{1}$ and $A_{3}$, decads of points on $\mathcal{C}$; the Jacobian set of this $g_{10}^{\mathrm{I}}$ consists, as did a Jacobian set on $H$, of 28 points. The singular conic $Z^{2}=0$, a member of the pencil, meets $\mathcal{C}$ at eight points both at $A_{1}$ and $A_{3}$; removing each of $A_{1}$ and $A_{3}$ three times leaves a decad consisting of two quintuple points each, as with $H$, contributing four to the Jacobian set. So 20 points remain, and these will be contact pentads for four of the conies.

Such a pentad invariant, with the conic and $\mathcal{C}$, under $\mathcal{D}$, must have one member on the axis of each of the five involutions in $\mathcal{D}$. Each axis, however, meets $\mathcal{C}$ in eight points; the four not belonging to contact pentads are found to coincide in pairs at nodes of $\mathcal{C}$. For example: one axis is $X=Y$ whose eight intersections with $\mathfrak{C}$ are pin-pointed by the identity.

$2000 x^{8}+2240 x^{6}+1024 x^{5}+360 x^{4}+1 \equiv\left(10 x^{2}+4 x+1\right)^{2}\left(20 x^{4}-16 x^{3}+28 x^{2}-8 x+1\right)$.

The zeros of the biquadratic factor on the right account for one member of each 
contact pentad; those of the repeated quadratic factor account for the two nodes on $X=Y$, whose (complex) coordinates can thus be given explicitly. A plane octavic of genus 5 with two triple points has to have $21-5-2.3=10$ further double points or their equivalent.

\section{REFERENCES}

[1] H. F. BAKer, Prineiples of geometry, Cambridge, vol. IV, 1925.

[2] W. L. EDGE, Humbert's plane sexties of genus 5, Proceedings of the Cambridge Philos:phical Society, 47 (1951), pp. 483-495.

[3] W. L. EDGE, Three plane sextics and their automorphisms, Canadian Journal of Mathematics, 21 (1969), pp. 1263-1278.

[4] W. L. EDGE, The prineipal chords of an elliptic quartic, Proceedings of the Royal Society of Edinburgh (A) 71 (1972), pp. 43-50.

[5] W. L. EDGE, The osculating spaces of a certain curve in [ $n$ ], Proceedings of the Edinburgh Mathematical Society (2) 19 (1974), pp. 39-44.

[6] W. L. EDGE, The chord locus of a certain curve in [n], Proceedings of the Royal Society of Edinburgh (A) 71 (1973), pp. 337-343.

[7] G. Salmon, $A$ treatise on the analytic geometry of three dimensions, 4th ed., Dublin, 1882.

[8] C. SEGRE, Introduzione alla geometria sopra un ente algebrico semplicemente infinito, Annali di matematica (2) 22 (1894), pp. 41-142; Opere, vol. I, Rome, 1957, pp. 198-304.

[9] F. Severi, Sopra alcune singolarità delle curve di un iperspazio, Memorie Accad. Scienze di Torino, 2 (1902), pp. 81-114.

[10] F. Severi, Lezioni di geometria algebrica, Padua, 1908.

[11] A. WIMAN, Über die algebraischen Curven von den Geschlectern $p=4,5$ und 6, welche eindeutige Transformationen in sich besitzen, Svenska, Vet-Akad. Handlingar, Bihang till Handlingar 21 (1895), afd. 1, no. 3, 41 pp.

[12] H. G. ZeUTHEN, Nouvelle démonstration de théorèmes sur des séries de points correspondants sur deux courbes, Mathematische Annalen, 3 (1871), pp. 150-156.

[13] H. G. Zeutmen, Lehrbuch der abzählenden Methoden der Geometrie, Teubner, Leipzig and Berlin, 1914. 